\title{
Dynamical replica analysis of processes on finitely connected random graphs: I. Vertex covering
}

\author{
A Mozeika and A C C Coolen \\ Department of Mathematics, King's College London, The Strand, London WC2R 2LS, UK \\ E-mail: alexander.mozeika@kcl.ac.uk and ton.coolen@kcl.ac.uk
}

Received 7 December 2007, in final form 5 February 2008

Published 4 March 2008

Online at stacks.iop.org/JPhysA/41/115003

\begin{abstract}
We study the stochastic dynamics of Ising spin models with random bonds, interacting on finitely connected Poissonian random graphs. We use the dynamical replica method to derive closed dynamical equations for the joint spin-field probability distribution, and solve these within the replica-symmetry ansatz. Although the theory is developed in a general setting, with a view to future applications in various other fields, in this paper we apply it mainly to the dynamics of the Glauber algorithm (extended with cooling schedules) when running on the so-called vertex cover optimization problem. Our theoretical predictions are tested against both Monte Carlo simulations and known results from equilibrium studies. In contrast to previous dynamical analyses based on deriving closed equations for only a small number of scalar order parameters, the agreement between theory and experiment in the present study is nearly perfect.
\end{abstract}

PACS numbers: $02.50 . E y$, 05.90.+m, 64.60.Cn, 89.20.Ff

\section{Introduction}

The interest in studying finitely connected (FC) spin systems on random graphs, as introduced in [1] more than 20 years ago, has grown in recent years. For this there appears to be at least two reasons. First, FC spin systems can be seen as an intermediate step between fully connected mean-field spin models [2] and finite-dimensional spin models. Although they are still of the mean-field type in the sense that random site permutations are irrelevant in the mean-field limit, the finite connectivity introduces notions of site neighbourhood, distances, etc. This attractive property has drawn many into this field, and we have by now achieved a thorough understanding of the equilibrium behaviour of FC spin systems [3-7]. Second, many optimization and decision problems in theoretical computer science can be mapped into the models of FC spin systems. This mapping allowed such problems to be studied with analytic 
methods of statistical mechanics, and has been very fruitful especially in the study of K-SAT $[8,9]$, vertex covering [10] and graph colouring [11, 12].

Although our understanding of the equilibrium properties of FC spin systems is now quite advanced, that of the non-equilibrium behaviour of such systems is, despite recent progress [13-19], still relatively limited in comparison. Dynamical studies are generally harder, by definition, as they incorporate the equilibrium state as a special case. In the domain of the dynamics of FC spin systems, the generating functional method (or the path integration technique) of [20] is the only exact method available today. There has been some success in applying this method to finitely connected soft spin systems [13, 14] and Ising spin systems [16]. However, the generating functional method leads one in FC systems to a formalism involving a rather complicated dynamical order parameter (describing the joint statistics of single-site spin 'paths' and single-site field perturbation 'paths') which is generally difficult to handle. Even for parallel dynamics [16] it is effectively equivalent to having a number of scalar order parameters that grows exponentially with the number of discrete time steps considered. For that reason, even in generating functional analysis studies one is in practice forced to make further approximations to tame this explosion of order parameters.

An alternative approach to the dynamics of FC spin systems is the dynamic replica theory (DRT) [21, 22], which was initially developed for fully connected systems. In contrast to generating functional analysis, DRT in its present form is not (yet) exact; however, one can increase its accuracy systematically by increasing the size of the chosen order parameter set [22]. The great advantage of DRT in the study of FC spin systems, compared to generating functional analysis, is that the effective number of order parameters does not grow with time. Recently, the DRT method [17] and its equivalent [15] were used to study the dynamics of FC Ising spin systems, but only for a relatively small number of dynamic order parameters. Although its performance on regular random graphs was found to be very good [17, 15], for random Poissonian graphs it was found to be quite poor [17]. In the present paper we develop the DRT method further, and cure the previous limitations by increasing the size of the order parameter set, following $[22,15]$, to the full joint spin-field distribution. We then demonstrate the performance of the resulting improved theory by application to the so-called minimal vertex cover problem [10] on Poissonian random graphs.

This paper is organized as follows. In section 2, we define our model and derive an exact dynamical equation for the joint spin-field probability distribution. In section 3, we close this equation using the standard assumptions and procedures of DRT. We simplify our dynamical theory by making the standard replica-symmetry ansatz in section 3.2. In section 4, we apply our resulting formalism to the dynamics of the Glauber algorithm, extended with simulated annealing-type cooling schedules, when running on the minimal vertex cover problem. The outcome of solving our dynamical equations numerically are compared to measurements taken in the Monte Carlo simulations. Finally, in section 6 we summarize and discuss our results.

\section{Model definitions and macroscopic laws}

We consider a system of $N$ Ising spins, $\sigma_{i} \in\{-1,1\}$, which are placed on the vertices of a random Erdös-Rényi graph [23]. Spins interact only when they are connected. Their microscopic dynamics are governed by a Glauber-type stochastic algorithm. At each iteration of this algorithm, a site $i$ is drawn randomly from the set $\{1, \ldots, N\}$ of all sites, and spin $\sigma_{i}$ is subsequently flipped with probability

$$
P\left(\sigma_{i} \rightarrow-\sigma_{i}\right)=\frac{1}{2}\left[1-\sigma_{i} \tanh \left[\beta h_{i}(\sigma)\right]\right]
$$


where $h_{i}$ is a local field, defined as

$$
h_{i}(\sigma)=\sum_{j \neq i} c_{i j} J_{i j} \sigma_{j}+\theta,
$$

with $\sigma=\left(\sigma_{1}, \ldots, \sigma_{N}\right)$. The inverse temperature $\beta=T^{-1}$ controls the level of noise in the system; the dynamics is fully random for $\beta=0$ and fully deterministic for $\beta \rightarrow \infty$. The parameter $\theta$ defines a uniform external field. The set of random variables $\left\{c_{i j} J_{i j}\right\}$ is regarded as a quenched disorder. The bonds $J_{i j}$ are symmetric, namely, $J_{i j}=J_{j i}$, and drawn independently from a probability distribution $P(J)$. The independently distributed random variables $c_{i j} \in\{0,1\}$ are the entries of a symmetric adjacency matrix with zeros on the main diagonal, defining the random graph. In this paper, we consider finitely connected (FC) random graphs of the Erdös-Rényi [23] type, where

$$
\forall i<j: \quad P\left(c_{i j}\right)=\frac{c}{N} \delta_{c_{i j}, 1}+\left(1-\frac{c}{N}\right) \delta_{c_{i j}, 0}
$$

with $c=O\left(N^{0}\right)$. In the $N \rightarrow \infty$ limit, the average number of connections per spin (or vertex) remains finite, and the distribution of connectivities (or vertex degrees) is given by a Poisson distribution with mean $c$ :

$$
P_{c}(k)=c^{k} \mathrm{e}^{-c} / k !
$$

Process (1) can be written in the form of a master equation for the evolution of the microscopic state probability in continuous time ${ }^{1}$,

$$
\frac{\mathrm{d}}{\mathrm{d} t} p_{t}(\boldsymbol{\sigma})=\sum_{i=1}^{N}\left[p_{t}\left(F_{i} \boldsymbol{\sigma}\right) w_{i}\left(F_{i} \boldsymbol{\sigma}\right)-p_{t}(\boldsymbol{\sigma}) w_{i}(\boldsymbol{\sigma})\right],
$$

in which $F_{i}$ is a spin-flip operator $F_{i} \Omega(\sigma)=\Omega\left(\sigma_{1}, \ldots,-\sigma_{i}, \ldots, \sigma_{N}\right)$, and the quantities $w_{i}(\sigma)$ are the transition rates given by

$$
w_{i}(\sigma)=\frac{1}{2}\left[1-\sigma_{i} \tanh \left[\beta h_{i}(\sigma)\right]\right] .
$$

This process evolves towards the equilibrium Boltzmann probability distribution $p_{\infty}(\sigma) \sim$ $\exp [-\beta H(\sigma)]$, with the Hamiltonian

$$
H(\boldsymbol{\sigma})=-\sum_{i<j} \sigma_{i} c_{i j} J_{i j} \sigma_{j}-\theta \sum_{i} \sigma_{i} .
$$

In general, it is not possible to solve the $2^{N}$ coupled equations (5) directly. Therefore, instead of following the evolution of the microscopic distribution $p_{t}(\sigma)$, one turns to alternative descriptions of the dynamics in terms of macroscopic observables.

For the reasons given in the introduction, we now follow the steps of the dynamic replica theory [22], and consider the evolution in time of an arbitrary set of $\ell$ macroscopic observables $\Omega(\sigma)=\left(\Omega_{1}(\sigma), \ldots, \Omega_{\ell}(\sigma)\right)$, where each individual $\Omega_{k}(\sigma)$ is taken to be of order $O\left(N^{0}\right)$. We derive a Kramers-Moyal expansion for the associated macroscopic probability distribution $P_{t}(\Omega)=\sum_{\sigma} \delta[\Omega-\Omega(\sigma)] p_{t}(\sigma)$ by inserting the master equation (5) into the time derivative of $P_{t}(\Omega)$, and expanding the result in powers of the 'discrete derivatives' $\Delta_{i}^{\mu}(\sigma)=\Omega_{\mu}\left(F_{i} \sigma\right)-\Omega_{\mu}(\sigma)$. This gives

$$
\begin{aligned}
\frac{\mathrm{d}}{\mathrm{d} t} P_{t}(\boldsymbol{\Omega})= & -\sum_{\mu=1}^{\ell} \frac{\partial}{\partial \Omega_{\mu}}\left\{P_{t}(\boldsymbol{\Omega})\left\langle\sum_{i} w_{i}(\boldsymbol{\sigma}) \Delta_{i}^{\mu}(\boldsymbol{\sigma})\right\rangle_{\Omega ; t}\right\} \\
& +\frac{1}{2} \sum_{\mu, \nu=1}^{\ell} \frac{\partial^{2}}{\partial \Omega_{\mu} \partial \Omega_{v}}\left\{P_{t}(\boldsymbol{\Omega})\left\langle\sum_{i} w_{i}(\boldsymbol{\sigma}) \Delta_{i}^{\mu}(\boldsymbol{\sigma}) \Delta_{i}^{v}(\boldsymbol{\sigma})\right\rangle_{\Omega ; t}\right\}+O\left(N \ell^{3} \Delta^{3}\right),
\end{aligned}
$$

1 This involves formally the introduction of random durations for the individual spin updates which are $N^{-1}$ on average, and which for finite $N$ are drawn from a specific distribution [24]. 
where we used the sub-shell (or conditional) average

$$
\langle f(\boldsymbol{\sigma})\rangle_{\boldsymbol{\Omega} ; t}=\frac{\sum_{\boldsymbol{\sigma}} p_{t}(\boldsymbol{\sigma}) \delta[\boldsymbol{\Omega}-\boldsymbol{\Omega}(\boldsymbol{\sigma})] f(\boldsymbol{\sigma})}{\sum_{\boldsymbol{\sigma}} p_{t}(\boldsymbol{\sigma}) \delta[\boldsymbol{\Omega}-\boldsymbol{\Omega}(\boldsymbol{\sigma})]} .
$$

If the diffusion term in expansion (8) vanishes for $N \rightarrow \infty$, then (8) acquires the Liouville form, the solution of which describes the following deterministic flow:

$$
\frac{\mathrm{d}}{\mathrm{d} t} \boldsymbol{\Omega}=\left\langle\sum_{i} w_{i}(\boldsymbol{\sigma})\left[\boldsymbol{\Omega}\left(F_{i} \boldsymbol{\sigma}\right)-\boldsymbol{\Omega}(\boldsymbol{\sigma})\right]\right\rangle_{\Omega ; t} .
$$

This is then exact for $N \rightarrow \infty$, but not necessarily closed, due to the presence of the microscopic probability $p_{t}(\boldsymbol{\sigma})$ in (9). In DRT, in order to close equation (10), one assumes equi-partitioning of probability within the $\Omega$ sub-shells, i.e. one takes $p_{t}(\sigma)$ to depend on $\zeta$ only through $\Omega(\sigma)$. The impact of this assumption on the accuracy of the theory depends critically on the choice of observables $\Omega(\sigma)$.

In this paper, our choice of observables $\Omega(\sigma)$ is, as in [22], the (infinite-dimensional) set given by the joint spin-field distribution

$$
D(s, h ; \sigma)=\frac{1}{N} \sum_{i} \delta_{s, \sigma_{i}} \delta\left[h-h_{i}(\boldsymbol{\sigma})\right] .
$$

We assume that this distribution (11) is well behaved in the sense that it can be evaluated first for a finite number $\ell$ of field arguments $h_{\mu}$, and that the limit $\ell \rightarrow \infty$ can be taken after the thermodynamic limit $N \rightarrow \infty$. For now on we thus have $2 \ell$ observables $D\left(s, h_{\mu} ; \sigma\right)$ with $\mu=1, \ldots, \ell$ and $s \in\{-1,1\}$. In order to compute (10) we must work out the discrete derivatives $\Delta_{i}^{s \mu}(\boldsymbol{\sigma})=D\left(s, h_{\mu} ; F_{i} \boldsymbol{\sigma}\right)-D\left(s, h_{\mu} ; \boldsymbol{\sigma}\right)$ :

$$
\begin{aligned}
\Delta_{i}^{s \mu}(\boldsymbol{\sigma})= & \frac{1}{N} \sum_{j} \delta_{s, F_{i} \sigma_{j}} \delta\left[h_{\mu}-h_{j}\left(F_{i} \boldsymbol{\sigma}\right)\right]-\frac{1}{N} \sum_{j} \delta_{s, \sigma_{j}} \delta\left[h_{\mu}-h_{j}(\boldsymbol{\sigma})\right] \\
= & \frac{1}{N} \sum_{j \neq i} \delta_{s, \sigma_{j}} c_{i j}\left\{\delta_{\sigma_{i}, 1} \delta\left[h_{\mu}-h_{j}(\sigma)+2 J_{i j}\right]+\delta_{\sigma_{i},-1} \delta\left[h_{\mu}-h_{j}(\sigma)-2 J_{i j}\right]\right. \\
& \left.-\delta\left[h_{\mu}-h_{j}(\sigma)\right]\right\}+\frac{1}{N}\left\{\delta_{s,-\sigma_{i}}-\delta_{s, \sigma_{i}}\right\} \delta\left[h_{\mu}-h_{i}(\boldsymbol{\sigma})\right] .
\end{aligned}
$$

Thus, $\Delta_{i}^{s \mu}(\sigma)=O\left(N^{-1}\right)$, so for $N \rightarrow \infty$ the diffusion term in (8) vanishes and the macroscopic observables $D\left(s, h_{\mu} ; \sigma\right)$ evolve deterministically according to (10). Inserting (12) into (10) gives us a diffusion equation for the joint spin-field distribution

$$
\begin{aligned}
\frac{\partial}{\partial t} D\left(s, h_{\mu}\right)= & \frac{1}{2}\left[1+s \tanh \left[\beta h_{\mu}\right]\right] D\left(-s, h_{\mu}\right)-\frac{1}{2}\left[1-s \tanh \left[\beta h_{\mu}\right]\right] D\left(s, h_{\mu}\right) \\
& +\frac{1}{2} \sum_{s^{\prime}} \int \mathrm{d} h^{\prime}\left[1-s^{\prime} \tanh \left[\beta h^{\prime}\right]\right] \\
& \times\left\langle\frac{1}{N} \sum_{i \neq j} \delta_{s^{\prime}, \sigma_{i}} \delta_{s, \sigma_{j}} c_{i j} \delta\left[h^{\prime}-h_{i}(\sigma)\right] \delta\left[h_{\mu}-h_{j}(\sigma)+2 J_{i j} s^{\prime}\right]\right\rangle_{D ; t} \\
& -\frac{1}{2} \sum_{s^{\prime}} \int \mathrm{d} h^{\prime}\left[1-s^{\prime} \tanh \left[\beta h^{\prime}\right]\right] \\
& \times\left\langle\frac{1}{N} \sum_{i \neq j} \delta_{s^{\prime}, \sigma_{i}} \delta_{s, \sigma_{j}} c_{i j} \delta\left[h^{\prime}-h_{i}(\sigma)\right] \delta\left[h_{\mu}-h_{j}(\sigma)\right]\right\rangle_{D ; t},
\end{aligned}
$$


with the sub-shell average

$$
\langle f(\boldsymbol{\sigma})\rangle_{D ; t}=\frac{\sum_{\boldsymbol{\sigma}} p_{t}(\boldsymbol{\sigma}) f(\boldsymbol{\sigma}) \prod_{s \mu} \delta\left[D\left(s, h_{\mu}\right)-D\left(s, h_{\mu} ; \boldsymbol{\sigma}\right)\right]}{\sum_{\sigma^{\prime}} p_{t}\left(\boldsymbol{\sigma}^{\prime}\right) \prod_{s \mu} \delta\left[D\left(s, h_{\mu}\right)-D\left(s, h_{\mu} ; \boldsymbol{\sigma}^{\prime}\right)\right]} .
$$

The non-trivial objects in (13) are the two averages, with angular brackets. To compute these efficiently we introduce the following kernel, where $\tilde{s} \in\left\{0, s^{\prime}\right\}$,

$$
\tilde{A}\left[s, s^{\prime} ; h, h^{\prime} ; \tilde{s}\right]=\left\langle\frac{1}{c N} \sum_{i j} \delta_{s^{\prime}, \sigma_{i}} \delta_{s, \sigma_{j}} c_{i j} \delta\left[h^{\prime}-h_{i}(\sigma)\right] \delta\left[h-h_{j}(\sigma)+2 J_{i j} \tilde{s}\right]\right\rangle_{D ; t} .
$$

For $\tilde{s}=0$, kernel (15) defines the joint spin-field probability of connected sites (a similar object was used to study the dynamics of the Ising ferromagnet on a regular random graph [15]). In the limit $N \rightarrow \infty$, definition (15) allows us to write (13) as

$$
\begin{aligned}
\frac{\partial}{\partial t} D(s, h)= & \frac{1}{2}[1+s \tanh [\beta h]] D(-s, h)-\frac{1}{2}[1-s \tanh [\beta h]] D(s, h) \\
& +\frac{1}{2} c \sum_{s^{\prime}} \int \mathrm{d} h^{\prime}\left[1-s^{\prime} \tanh \left[\beta h^{\prime}\right]\right] \tilde{A}\left[s, s^{\prime} ; h, h^{\prime} ; s^{\prime}\right] \\
& -\frac{1}{2} c \sum_{s^{\prime}} \int \mathrm{d} h^{\prime}\left[1-s^{\prime} \tanh \left[\beta h^{\prime}\right]\right] \tilde{A}\left[s, s^{\prime} ; h, h^{\prime} ; 0\right] .
\end{aligned}
$$

This dynamical equation (16) is exact for large $N$, but not yet closed. Closure requires eliminating $p_{t}(\sigma)$ from (15).

\section{Replica analysis of the dynamics}

\subsection{Closure and disorder averaging}

To evaluate the right-hand side of (16) we make the usual assumptions of the dynamic replica method. The observables $D\left(s, h_{\mu} ; \sigma\right)$ are taken to be self-averaging with respect to the disorder at any time, i.e. to depend only on the statistics of the $\left\{c_{i j} J_{i j}\right\}$ rather than their realization. Second, we assume equi-partitioning of the microscopic probability within the $D\left(s, h_{\mu} ; \sigma\right)$ sub-shells of the conditional average (14). These assumptions and the equivalence of sites after disorder averaging lead us to

$$
\begin{aligned}
A\left[s, s^{\prime} ; h, h^{\prime} ; \tilde{s}\right] & =\lim _{N \rightarrow \infty} \frac{N-1}{c}\left\langle\frac{\sum_{\sigma} \prod_{\tau \mu} \delta\left[D\left(\tau, h_{\mu}\right)-D\left(\tau, h_{\mu} ; \boldsymbol{\sigma}\right)\right]}{\sum_{\sigma^{\prime}} \prod_{\tau \mu} \delta\left[D\left(\tau, h_{\mu}\right)-D\left(\tau, h_{\mu} ; \boldsymbol{\sigma}^{\prime}\right)\right]}\right. \\
& \left.\times \delta_{s^{\prime}, \sigma_{1}} \delta_{s, \sigma_{2}} c_{12} \delta\left[h^{\prime}-h_{1}(\boldsymbol{\sigma})\right] \delta\left[h-h_{2}(\boldsymbol{\sigma})+2 J_{12} \tilde{s}\right]\right\rangle_{\left\{c_{i j} J_{i j}\right\}} .
\end{aligned}
$$

We eliminate the fraction from the above expression via the replica identity

$$
\frac{\sum_{\boldsymbol{\sigma}} \Phi(\boldsymbol{\sigma}) W(\boldsymbol{\sigma})}{\sum_{\boldsymbol{\sigma}} W(\boldsymbol{\sigma})}=\lim _{n \rightarrow 0} \sum_{\boldsymbol{\sigma}^{1}} \cdots \sum_{\boldsymbol{\sigma}^{n}} \Phi\left(\boldsymbol{\sigma}^{1}\right) \prod_{\alpha=1}^{n} W\left(\boldsymbol{\sigma}^{\alpha}\right)
$$

which leads to

$$
\begin{aligned}
A\left[s, s^{\prime} ; h, h^{\prime} ; \tilde{s}\right] & =\lim _{N \rightarrow \infty} \lim _{n \rightarrow 0} \frac{N-1}{c}\left\langle\sum_{\sigma^{1}} \cdots \sum_{\sigma^{n}}\right. \\
& \times \delta_{s^{\prime}, \sigma_{1}^{1}} \delta_{s, \sigma_{2}^{1}} c_{12} \delta\left[h^{\prime}-h_{1}\left(\sigma^{1}\right)\right] \delta\left[h-h_{2}\left(\sigma^{1}\right)+2 J_{12} \tilde{s}\right] \\
& \left.\times \prod_{\alpha=1}^{n} \prod_{\tau \mu} \delta\left[D\left(\tau, h_{\mu}\right)-\frac{1}{N} \sum_{i} \delta_{\tau, \sigma_{i}^{\alpha}} \delta\left[h_{\mu}-h_{i}\left(\sigma^{\alpha}\right)\right]\right]\right\rangle_{\left\{c_{i j} J_{i j}\right\}} .
\end{aligned}
$$


We can remove the disorder-dependent local fields $\left\{h_{i}\left(\sigma^{\alpha}\right)\right\}$ from inside the delta functions by inserting into (19) the following integral representation of unity: $1=\int \prod_{\alpha i} \mathrm{~d} H_{i}^{\alpha} \delta\left[H_{i}^{\alpha}-\right.$ $\left.h_{i}\left(\sigma^{\alpha}\right)\right]$. Writing the latter delta functions in integral form gives

$$
\begin{aligned}
A\left[s, s^{\prime} ; h, h^{\prime} ; \tilde{s}\right] & =\lim _{N \rightarrow \infty} \lim _{n \rightarrow 0} \frac{N-1}{c} \sum_{\sigma^{1}} \cdots \sum_{\sigma^{n}} \int \prod_{\alpha i}\left[\mathrm{~d} H_{i}^{\alpha} \mathrm{d} \hat{h}_{i}^{\alpha} \exp \left[\mathrm{i} \hat{h}_{i}^{\alpha} H_{i}^{\alpha}\right]\right] \\
& \times \prod_{\alpha=1}^{n} \prod_{\tau \mu} \delta\left[D\left(\tau, h_{\mu}\right)-\frac{1}{N} \sum_{i} \delta_{\tau, \sigma_{i}^{\alpha}} \delta\left[h_{\mu}-H_{i}^{\alpha}\right]\right] \\
& \times \delta_{s^{\prime}, \sigma_{1}^{1}} \delta_{s, \sigma_{2}^{1}} \delta\left[h^{\prime}-H_{1}^{1}\right]\left\langle c_{12} \delta\left[h-H_{2}^{1}+2 J_{12} \tilde{s}\right] \mathrm{e}^{-\mathrm{i} \sum_{\alpha i} \hat{h}_{i}^{\alpha} h_{i}\left(\sigma^{\alpha}\right)}\right\rangle_{\left\{c_{i j} J_{i j}\right\}} .
\end{aligned}
$$

After the average over the disorder is taken (see appendix A for details), we then find

$$
\begin{aligned}
A\left[s, s^{\prime} ; h, h^{\prime} ; \tilde{s}\right] & =\lim _{N \rightarrow \infty} \lim _{n \rightarrow 0} \sum_{\sigma^{1}} \cdots \sum_{\sigma^{n}} \delta_{s^{\prime}, \sigma_{1}^{1}} \delta_{s, \sigma_{2}^{1}} \int \prod_{\alpha i}\left[\frac{\mathrm{d} H_{i}^{\alpha} \mathrm{d} \hat{h}_{i}^{\alpha}}{2 \pi} \mathrm{e}^{\mathrm{i} \hat{h}_{i}^{\alpha} H_{i}^{\alpha}}\right] \\
& \times \delta\left[h^{\prime}-H_{1}^{1}\right] \prod_{\tau \mu \alpha} \delta\left[D\left(\tau, h_{\mu}\right)-\frac{1}{N} \sum_{i} \delta_{\left.\tau, \sigma_{i}^{\alpha} \delta\left[h_{\mu}-H_{i}^{\alpha}\right]\right]}\right. \\
& \times \mathrm{e}^{-\mathrm{i} \sum_{\alpha i} \hat{h}_{i}^{\alpha} \theta} \int \mathrm{d} J P(J) \delta\left[h-H_{2}^{1}+2 J \tilde{s}\right] \mathrm{e}^{\left.-\mathrm{i} J \sum_{\alpha} \hat{h}_{1}^{\alpha} \sigma_{2}^{\alpha}+\hat{h}_{2}^{\alpha} \sigma_{1}^{\alpha}\right]} \\
& \times \exp \left[\frac{c}{2 N} \sum_{i j}\left(\int \mathrm{d} J P(J) \mathrm{e}^{-\mathrm{i} J \sum_{\alpha}\left[\hat{h}_{i}^{\alpha} \sigma_{j}^{\alpha}+\hat{h}_{j}^{\alpha} \sigma_{i}^{\alpha}\right]}-1\right)+O(1)\right] .
\end{aligned}
$$

The $\mathcal{O}(1)$ term in the exponent of the last line is independent of $\left\{s, s^{\prime}, h, h^{\prime}, \tilde{s}\right\}$, and can always be recovered from the normalization $\sum_{s, s^{\prime}} \int \mathrm{d} h \mathrm{~d} h^{\prime} A\left[s, s^{\prime} ; h, h^{\prime} ; \tilde{s}\right]=1$. Next, we achieve factorization over sites in (21) upon isolating the density

$$
P\left(\boldsymbol{\sigma}, \hat{\boldsymbol{h}} ;\left\{\boldsymbol{\sigma}_{i}\right\},\left\{\boldsymbol{h}_{i}\right\}\right)=\frac{1}{N} \sum_{i} \delta_{\sigma, \sigma_{i}} \delta\left[\hat{\boldsymbol{h}}-\hat{\boldsymbol{h}}_{i}\right],
$$

where $\boldsymbol{\sigma}=\left(\sigma_{1}, \ldots, \sigma_{n}\right), \boldsymbol{\sigma}_{i}=\left(\sigma_{i}^{1}, \ldots, \sigma_{i}^{n}\right)$ and similarly for the replicated vectors $\hat{\boldsymbol{h}}$, etc, via insertion into (21) of the $\delta$-functional unity representation

$$
1=\int \prod_{\sigma \hat{\boldsymbol{h}}} \mathrm{d} P(\boldsymbol{\sigma}, \hat{\boldsymbol{h}}) \delta\left[P(\boldsymbol{\sigma}, \hat{\boldsymbol{h}})-P\left(\boldsymbol{\sigma}, \hat{\boldsymbol{h}} ;\left\{\boldsymbol{\sigma}_{i}\right\},\left\{\boldsymbol{h}_{i}\right\}\right)\right]
$$

which gives, with the short-hands $\langle g(J)\rangle_{J}=\int \mathrm{d} J P(J) g(J)$ and $\mathbf{x} \cdot \mathbf{y}=\sum_{\alpha} x^{\alpha} y^{\alpha}$,

$$
\begin{aligned}
A\left[s, s^{\prime} ; h, h^{\prime} ; \tilde{s}\right] & =\lim _{N \rightarrow \infty} \lim _{n \rightarrow 0} \int \prod_{\tau \mu \alpha}\left[\frac{\mathrm{d} \hat{D}_{\alpha}\left(\tau, h_{\mu}\right)}{2 \pi / N}\right] \int \prod_{\sigma \hat{\boldsymbol{h}}}\left[\frac{\mathrm{d} \hat{P}(\sigma, \hat{\boldsymbol{h}}) \mathrm{d} P(\boldsymbol{\sigma}, \hat{\boldsymbol{h}})]}{2 \pi / N}\right] \\
& \times \exp \left\{N \left[\mathrm{i} \sum_{\tau \mu \alpha} \hat{D}_{\alpha}\left(\tau, h_{\mu}\right) D\left(\tau, h_{\mu}\right)+\mathrm{i} \sum_{\sigma \hat{\boldsymbol{h}}} \hat{P}(\boldsymbol{\sigma}, \hat{\boldsymbol{h}}) P(\boldsymbol{\sigma}, \hat{\boldsymbol{h}})+O\left(\frac{1}{N}\right)\right.\right. \\
& \left.\left.+\frac{1}{2} c \sum_{\sigma \sigma^{\prime}} \int \mathrm{d} \hat{\boldsymbol{h}} \mathrm{d} \hat{\boldsymbol{h}}^{\prime} P(\boldsymbol{\sigma}, \hat{\boldsymbol{h}}) P\left(\boldsymbol{\sigma}^{\prime}, \hat{\boldsymbol{h}}^{\prime}\right)\left\langle\mathrm{e}^{-\mathrm{i} J\left[\hat{\boldsymbol{h}} \cdot \boldsymbol{\sigma}^{\prime}+\hat{\boldsymbol{h}}^{\prime} \cdot \boldsymbol{\sigma}\right]}-1\right\rangle_{J}\right]\right\} \\
& \times \sum_{\sigma^{1}} \cdots \sum_{\sigma^{n}} \int \prod_{i}\left[\frac{\mathrm{d} \boldsymbol{H}_{i} \mathrm{~d} \hat{\boldsymbol{h}}_{i}}{2 \pi} \mathrm{e}^{\mathrm{i} \hat{\boldsymbol{h}}_{i} \cdot\left[\boldsymbol{H}_{i}-\theta\right]}\right]
\end{aligned}
$$

6 


$$
\begin{aligned}
& \times \mathrm{e}^{-\mathrm{i} \sum_{\tau \mu \alpha} \hat{D}_{\alpha}\left(\tau, h_{\mu}\right) \sum_{i} \delta_{\tau, \sigma_{i}^{\alpha}} \delta\left[h_{\mu}-H_{i}^{\alpha}\right]-\mathrm{i} \sum_{\sigma} \hat{\boldsymbol{h}} \hat{P}(\sigma, \hat{\boldsymbol{h}}) \sum_{i} \delta_{\sigma, \sigma_{i}} \delta\left[\hat{\boldsymbol{h}}-\hat{\boldsymbol{h}}_{i}\right]} \\
& \times \delta_{s^{\prime}, \sigma_{1}^{1}} \delta_{s, \sigma_{2}^{1}} \delta\left[h^{\prime}-H_{1}^{1}\right]\left\langle\delta\left[h-H_{2}^{1}+2 J \tilde{s}\right] \mathrm{e}^{-\mathrm{i} J\left[\hat{\boldsymbol{h}}_{1} \cdot \sigma_{2}+\hat{\boldsymbol{h}}_{2} \cdot \sigma_{1}\right]}\right\rangle_{J} .
\end{aligned}
$$

We rescale the conjugate integration variables according to $\hat{P}(\sigma, \hat{\boldsymbol{h}}) \rightarrow \mathrm{d} \hat{\boldsymbol{h}} \hat{P}(\sigma, \hat{\boldsymbol{h}})$, which converts the sum over $\hat{\boldsymbol{h}}$ in the exponent of (24) into a well-defined integral when $\mathrm{d} \hat{\boldsymbol{h}} \rightarrow \mathbf{0}$, and $\hat{D}_{\alpha}\left(\tau, h_{\mu}\right) \rightarrow \Delta h_{\mu} \hat{D}_{\alpha}\left(\tau, h_{\mu}\right)$. We write the resulting path integral measure as $\{\mathrm{d} P \mathrm{~d} \hat{P} \mathrm{~d} \hat{D}\}$. Next, we define an effective single-site measure $M$ :

$$
\begin{aligned}
\langle f[\boldsymbol{H}, \hat{\boldsymbol{h}} ; \boldsymbol{\sigma}]\rangle_{M} & =\frac{\sum_{\boldsymbol{\sigma}} \int \mathrm{d} \boldsymbol{H} \mathrm{d} \hat{\boldsymbol{h}} M[\boldsymbol{H}, \hat{\boldsymbol{h}}, \boldsymbol{\sigma} \mid \theta] f[\boldsymbol{H}, \hat{\boldsymbol{h}} ; \boldsymbol{\sigma}]}{\sum_{\boldsymbol{\sigma}} \int \mathrm{d} \boldsymbol{H} \mathrm{d} \hat{\boldsymbol{h}} M[\boldsymbol{H}, \hat{\boldsymbol{h}}, \boldsymbol{\sigma} \mid \theta]}, \\
M[\boldsymbol{H}, \hat{\boldsymbol{h}}, \boldsymbol{\sigma} \mid \theta] & =\mathrm{e}^{\mathrm{i} \hat{\boldsymbol{h}} \cdot[\boldsymbol{H}-\boldsymbol{\theta}]-\mathrm{i} \sum_{s \mu \alpha} \Delta h_{\mu} \hat{D}_{\alpha}\left(s, h_{\mu}\right) \delta_{s, \sigma_{\alpha}} \delta\left[h_{\mu}-H_{\alpha}\right]-\mathrm{i} \hat{P}(\boldsymbol{\sigma}, \hat{\boldsymbol{h}})}
\end{aligned}
$$

and the function

$$
\begin{aligned}
\Psi[\{P, \hat{P}, \hat{D}\}] & =\mathrm{i} \sum_{s \mu \alpha} \Delta h_{\mu} \hat{D}_{\alpha}\left(s, h_{\mu}\right) D\left(s, h_{\mu}\right)+\mathrm{i} \sum_{\sigma} \int \mathrm{d} \hat{\boldsymbol{h}} \hat{P}(\boldsymbol{\sigma}, \hat{\boldsymbol{h}}) P(\boldsymbol{\sigma}, \hat{\boldsymbol{h}}) \\
& +\log \sum_{\sigma} \int \mathrm{d} \boldsymbol{H} \mathrm{d} \hat{\boldsymbol{h}} M[\boldsymbol{H}, \hat{\boldsymbol{h}}, \boldsymbol{\sigma} \mid \theta] \\
& +\frac{1}{2} c \sum_{\sigma \sigma^{\prime}} \int \mathrm{d} \hat{\boldsymbol{h}} \mathrm{d} \hat{\boldsymbol{h}}^{\prime} P(\boldsymbol{\sigma}, \hat{\boldsymbol{h}}) P\left(\boldsymbol{\sigma}^{\prime}, \hat{\boldsymbol{h}}^{\prime}\right)\left\langle\mathrm{e}^{-\mathrm{i} J\left[\hat{\boldsymbol{h}} \cdot \boldsymbol{\sigma}^{\prime}+\hat{\boldsymbol{h}}^{\prime} \cdot \boldsymbol{\sigma}\right]}-1\right\rangle_{J} .
\end{aligned}
$$

Using these definitions and changing the order of the limits $N \rightarrow \infty$ and $n \rightarrow 0$ allows us to write (24) in the form

$$
\begin{aligned}
A\left[s, s^{\prime} ; h, h^{\prime} ; \tilde{s}\right] & =\lim _{n \rightarrow 0} \lim _{N \rightarrow \infty} \int\{\mathrm{d} P \mathrm{~d} \hat{P} \mathrm{~d} \hat{D}\} \mathrm{e}^{N \Psi[\{P, \hat{P}, \hat{D}\}]+O(1)} \\
& \times\left\langle\delta_{s^{\prime}, \sigma_{1}} \delta_{s, \sigma_{1}^{\prime}} \delta\left[h^{\prime}-H_{1}\right] \delta\left[h-H_{1}^{\prime}+2 J \tilde{s}\right] \mathrm{e}^{-\mathrm{i} J\left[\hat{\boldsymbol{h}} \cdot \sigma^{\prime}+\hat{\boldsymbol{h}}^{\prime} \cdot \sigma\right.}\right\rangle_{J, M, M^{\prime}},
\end{aligned}
$$

where the average $\langle\ldots\rangle_{M^{\prime}}$ corresponds to $\{\boldsymbol{H}, \hat{\boldsymbol{h}} ; \boldsymbol{\sigma}\} \rightarrow\left\{\boldsymbol{H}^{\prime}, \hat{\boldsymbol{h}}^{\prime} ; \boldsymbol{\sigma}^{\prime}\right\}$ in the definition of effective single-site measure (25). Finally, with the help of the normalization identity $\sum_{s s^{\prime}} \int \mathrm{d} h \mathrm{~d} h^{\prime} A\left[s, s^{\prime} ; h, h^{\prime} ; \tilde{s}\right]=1$, we compute (27) by the steepest descent:

$$
A\left[s, s^{\prime} ; h, h^{\prime} ; \tilde{s}\right]=\lim _{n \rightarrow 0} \frac{\left\langle\delta_{s^{\prime}, \sigma_{1}} \delta_{s, \sigma_{1}^{\prime}} \delta\left[h^{\prime}-H_{1}\right] \delta\left[h-H_{1}^{\prime}+2 J \tilde{s}\right] \mathrm{e}^{-\mathrm{i} J\left[\hat{\boldsymbol{h}} \cdot \boldsymbol{\sigma}^{\prime}+\hat{\boldsymbol{h}}^{\prime} \cdot \sigma\right]}\right\rangle_{J, M, M^{\prime}}}{\left\langle\mathrm{e}^{-\mathrm{i} J\left[\hat{\boldsymbol{h}} \cdot \boldsymbol{\sigma}^{\prime}+\hat{\boldsymbol{h}}^{\prime} \cdot \sigma\right]}\right\rangle_{J, M, M^{\prime}}},
$$

where $\{P, \hat{P}, \hat{D}\}$ are determined by extremization of $\Psi$. The functional variation of $\Psi$ with respect to $P(\sigma, \hat{\boldsymbol{h}}), \hat{P}(\sigma, \hat{\boldsymbol{h}})$ and $\hat{D}_{\alpha}\left(s, h_{\mu}\right)$ leads to the stationarity conditions

$$
\begin{aligned}
& D(s, h)=\left\langle\delta_{s, \sigma_{\alpha}} \delta\left[h-H_{\alpha}\right]\right\rangle_{M}, \\
& P(\boldsymbol{\sigma}, \hat{\boldsymbol{h}})=\left\langle\delta_{\sigma, \sigma^{\prime}} \delta\left[\hat{\boldsymbol{h}}-\hat{\boldsymbol{h}}^{\prime}\right]\right\rangle_{M^{\prime}}, \\
& \hat{P}(\boldsymbol{\sigma}, \hat{\boldsymbol{h}})=\mathrm{i} c \sum_{\sigma^{\prime}} \int \mathrm{d} \hat{\boldsymbol{h}}^{\prime} P\left(\boldsymbol{\sigma}^{\prime}, \hat{\boldsymbol{h}}^{\prime}\right)\left\langle\mathrm{e}^{-\mathrm{i} J\left[\hat{\boldsymbol{h}} \cdot \boldsymbol{\sigma}^{\prime}+\hat{\boldsymbol{h}}^{\prime} \cdot \boldsymbol{\sigma}\right]}-1\right\rangle_{J} .
\end{aligned}
$$

The conjugate order parameters $\hat{D}_{\alpha}(s, h)$ and $\hat{P}(\sigma, \hat{\boldsymbol{h}})$ are seen to play the role of Lagrange multipliers, ensuring normalization of $D(s, h)$ and $P(\sigma, \hat{h})$. The physical meaning of the density $P(\sigma, \hat{\boldsymbol{h}})$ is not yet clear, due to the presence of the vector $\hat{\boldsymbol{h}}$. 
We use equation (31) to eliminate the conjugate order parameters $\hat{P}(\sigma, \hat{\boldsymbol{h}})$ from the measure $M$. We assume that $\hat{D}(s, h)$ is sufficiently smooth in $h$, such that $\sum_{\mu} \Delta h_{\mu} \hat{D}_{\alpha}\left(s, h_{\mu}\right) f\left(h_{\mu}\right) \rightarrow \int \mathrm{d} H \hat{D}_{\alpha}(s, H) f(H)$ for $\ell \rightarrow \infty$. This leads to

$$
\begin{aligned}
M[\boldsymbol{H}, \hat{\boldsymbol{h}}, \boldsymbol{\sigma} \mid \theta] & =\exp \left\{\mathrm{i} \hat{\boldsymbol{h}} \cdot[\boldsymbol{H}-\boldsymbol{\theta}]-\mathrm{i} \sum_{\alpha} \hat{D}_{\alpha}\left(\sigma_{\alpha}, H_{\alpha}\right)\right. \\
& \left.+c \sum_{\boldsymbol{\sigma}^{\prime}} \int \mathrm{d} \hat{\boldsymbol{h}}^{\prime} P\left(\boldsymbol{\sigma}^{\prime}, \hat{\boldsymbol{h}}^{\prime}\right)\left\langle\mathrm{e}^{-\mathrm{i} J\left[\hat{\boldsymbol{h}}^{\prime} \cdot \boldsymbol{\sigma}^{\prime}+\hat{\boldsymbol{h}}^{\prime} \cdot \boldsymbol{\sigma}\right]}-1\right\rangle_{J}\right\}
\end{aligned}
$$

in the definition of the measure $M(25)$. The replica method requires finally that we take the $n \rightarrow 0$ limit in equations (28)-(30). To do this we need to make appropriate ansätze for the density $P(\sigma, \hat{h})$ and for the conjugate order parameters $\hat{D}_{\alpha}(s, H)$.

\subsection{Replica symmetry}

We evaluate (28)-(30) upon assuming ergodicity, which translates mathematically into the so-called replica-symmetry (RS) ansatz. First, the order parameters $\hat{D}_{\alpha}(s, H)$ depend only on a single replica index and are expected to be imaginary, so we put

$$
\hat{D}_{\alpha}(s, H)=\mathrm{i} \log d(s, H) .
$$

Second, the density $P(\sigma, \hat{\boldsymbol{h}})$ depends on a discrete and continuous vector in replica space. The RS ansatz demands its invariance under any joint permutation of their indices, which implies [7] that it must be of the general form

$$
P_{\mathrm{RS}}(\boldsymbol{\sigma}, \hat{\boldsymbol{h}})=\int\{\mathrm{d} P\} W[\{P\}] \prod_{\alpha=1}^{n} P\left(\sigma_{\alpha}, \hat{h}_{\alpha}\right),
$$

where $W[\{P\}]$ is a normalized functional distribution, i.e. $\int\{\mathrm{d} P\} W[\{P\}]=1$. The RS ansatz (33), (34), via its implications for the effective measure (25), will enable us to take the replica limit $n \rightarrow 0$ in equations (28)-(30). We insert (33), (34) into (32) and subsequently expand the exponential function containing $P_{\mathrm{RS}}(\sigma, \hat{\boldsymbol{h}})$, leading to

$$
\begin{aligned}
M_{\mathrm{RS}}[\boldsymbol{H}, \hat{\boldsymbol{h}}, \boldsymbol{\sigma} \mid \theta] & =\sum_{k \geqslant 0} \frac{c^{k}}{k !} \mathrm{e}^{-c} \int \prod_{\ell=1}^{k}\left\{\mathrm{~d} J_{\ell} P\left(J_{\ell}\right)\left\{\mathrm{d} P_{\ell}\right\} W\left[\left\{P_{l}\right\}\right]\right\} \\
\times & \prod_{\alpha=1}^{n}\left\{d\left(\sigma_{\alpha}, H_{\alpha}\right) \mathrm{e}^{\mathrm{i} \hat{h}_{\alpha}\left[H_{\alpha}-\theta\right]} \prod_{\ell=1}^{k}\left[\sum_{\sigma_{\ell}^{\alpha}} \int \mathrm{d} \hat{h}_{\ell}^{\alpha} P_{\ell}\left(\sigma_{\ell}^{\alpha}, \hat{h}_{\ell}^{\alpha}\right) \mathrm{e}^{-\mathrm{i} J_{\ell}\left[\hat{h}_{\alpha} \sigma_{\ell}^{\alpha}+\hat{h}_{\ell}^{\alpha} \sigma_{\alpha}\right]}\right]\right\} .
\end{aligned}
$$

We write averages with respect to the RS measure (35) as

$$
\langle f[\boldsymbol{H}, \hat{\boldsymbol{h}} ; \boldsymbol{\sigma}]\rangle_{M_{\mathrm{RS}}}=\frac{1}{M_{\mathrm{RS}}^{n}} \sum_{\sigma} \int \mathrm{d} \boldsymbol{H} \mathrm{d} \hat{\boldsymbol{h}} M_{\mathrm{RS}}[\boldsymbol{H}, \hat{\boldsymbol{h}}, \boldsymbol{\sigma} \mid \theta] f[\boldsymbol{H}, \hat{\boldsymbol{h}} ; \boldsymbol{\sigma}],
$$

where we defined the normalization constant $M_{\mathrm{RS}}^{n}=\sum_{\sigma} \int \mathrm{d} \boldsymbol{H} \mathrm{d} \hat{\boldsymbol{h}} M_{\mathrm{RS}}[\boldsymbol{H}, \hat{\boldsymbol{h}}, \boldsymbol{\sigma} \mid \theta]$. Clearly $\lim _{n \rightarrow 0} M_{\mathrm{RS}}^{n}=1$. We use the above results to solve equation (30) for the functional distribution $W[\{P\}]$, upon substituting the various RS expressions: 


$$
\begin{aligned}
& M_{\mathrm{RS}}^{n} \int\{\mathrm{d} P\} W[\{P\}] \prod_{\alpha=1}^{n} P\left(\sigma_{\alpha}, \hat{h}_{\alpha}\right)=\sum_{k \geqslant 0} \frac{c^{k}}{k !} \mathrm{e}^{-c} \int \prod_{\ell=1}^{k}\left\{\mathrm{~d} J_{\ell} P\left(J_{\ell}\right)\left\{\mathrm{d} P_{\ell}\right\} W\left[\left\{P_{\ell}\right\}\right]\right\} \\
& \times \prod_{\alpha=1}^{n} \int \mathrm{d} H_{\alpha} d\left(\sigma_{\alpha}, H_{\alpha}\right) \mathrm{e}^{\mathrm{i} \hat{h}_{\alpha}\left[H_{\alpha}-\theta\right]} \prod_{\ell=1}^{k}\left[\sum_{\sigma_{\ell}^{\alpha}} \int \mathrm{d} \hat{h}_{\ell}^{\alpha} P_{\ell}\left(\sigma_{\ell}^{\alpha}, \hat{h}_{\ell}^{\alpha}\right) \mathrm{e}^{-\mathrm{i} J_{\ell}\left[\hat{h}_{\alpha} \sigma_{\ell}^{\alpha}+\hat{h}_{\ell}^{\alpha} \sigma_{\alpha}\right]}\right] \\
& =\int\{\mathrm{d} P\} \prod_{\alpha=1}^{n} P\left(\sigma_{\alpha}, \hat{h}_{\alpha}\right) \sum_{k \geqslant 0} \frac{c^{k}}{k !} \mathrm{e}^{-c} \int \prod_{\ell=1}^{k}\left\{\mathrm{~d} J_{\ell} P\left(J_{\ell}\right)\left\{\mathrm{d} P_{\ell}\right\} W\left[\left\{P_{\ell}\right\}\right]\right\} \\
& \times Z^{n}\left[\left\{P_{1}, \ldots, P_{k}\right\}\right] \\
& \times \prod_{\sigma \hat{h}} \delta\left[P(\sigma, \hat{h})-\frac{\int \mathrm{d} H d(\sigma, H) \mathrm{e}^{\mathrm{i} \hat{h}[H-\theta]} \prod_{\ell=1}^{k}\left\{\sum_{\sigma_{\ell}} \int \mathrm{d} \hat{h}_{\ell} P_{\ell}\left(\sigma_{\ell}, \hat{h}_{\ell}\right) \mathrm{e}^{-\mathrm{i} J_{\ell}\left[\hat{h} \sigma_{\ell}+\hat{h}_{\ell} \sigma\right]}\right\}}{Z\left[\left\{P_{1}, \ldots, P_{k}\right\}\right]}\right],
\end{aligned}
$$

where

$$
\begin{aligned}
Z\left[\left\{P_{1}, \ldots, P_{k}\right\}\right]= & \sum_{\sigma} \int \mathrm{d} H \mathrm{~d} \hat{h} d(\sigma, H) \mathrm{e}^{\mathrm{i} \hat{h}[H-\theta]} \\
& \times \prod_{\ell=1}^{k}\left\{\sum_{\sigma_{\ell}} \int \mathrm{d} \hat{h}_{\ell} P_{\ell}\left(\sigma_{\ell}, \hat{h}_{\ell}\right) \mathrm{e}^{-\mathrm{i} J_{l}\left[\hat{h} \sigma_{\ell}+\hat{h}_{\ell} \sigma\right]}\right\} \\
= & 2 \pi \sum_{\sigma} \prod_{\ell=1}^{k}\left\{\sum_{\sigma_{\ell}} \int \mathrm{d} \hat{h}_{\ell} P_{\ell}\left(\sigma_{\ell}, \hat{h}_{\ell}\right) \mathrm{e}^{-\mathrm{i} J_{\ell} \hat{h}_{\ell} \sigma}\right\} d\left(\sigma, \sum_{\ell=1}^{k} J_{\ell} \sigma_{\ell}+\theta\right) .
\end{aligned}
$$

In the limit $n \rightarrow 0$, both the normalization term $Z^{n}\left[\left\{P_{1}, \ldots, P_{k}\right\}\right]$ and the constant $M_{\mathrm{RS}}^{n}$ reduce to unity, and we find an equation for the functional distribution $W[\{P\}]$ :

$$
\begin{aligned}
& W[\{P\}]=\sum_{k \geqslant 0} \frac{c^{k}}{k !} \mathrm{e}^{-c} \int \prod_{\ell=1}^{k}\left\{\mathrm{~d} J_{\ell} P\left(J_{\ell}\right)\left\{\mathrm{d} P_{\ell}\right\} W\left[\left\{P_{\ell}\right\}\right]\right\} \\
& \times \prod_{\sigma \hat{h}} \delta\left[P(\sigma, \hat{h})-\frac{\int \mathrm{d} H d(\sigma, H) \mathrm{e}^{\mathrm{i} \hat{h}[H-\theta]} \prod_{\ell=1}^{k}\left\{\sum_{\sigma_{\ell}} \int \mathrm{d} \hat{h}_{\ell} P_{\ell}\left(\sigma_{\ell}, \hat{h}_{\ell}\right) \mathrm{e}^{-\mathrm{i} J_{\ell}\left[\hat{h} \sigma_{\ell}+\hat{h}_{\ell} \sigma\right]}\right\}}{Z\left[\left\{P_{1}, \ldots, P_{k}\right\}\right]}\right] .
\end{aligned}
$$

In a similar fashion (see appendix B for details), we can compute the probability distributions $D(s, h)$ and $A\left[s, s^{\prime} ; h, h^{\prime} ; \tilde{s}\right]$ in RS ansatz. To compactify our formulae we define the Fourier transforms $\hat{P}(\sigma \mid x)=\int \mathrm{d} \hat{h} P(\sigma, \hat{h}) \mathrm{e}^{-\mathrm{i} \hat{h} x}$ (these should not be confused with the conjugate order parameter $\hat{P}(\sigma, \hat{h})$ which was eliminated in the previous section), in terms of which we find

$$
\begin{aligned}
D(s, h)=d(s, h) & \sum_{k \geqslant 0} \frac{c^{k}}{k !} \mathrm{e}^{-c} \int \prod_{\ell=1}^{k}\left\{\mathrm{~d} J_{\ell} P\left(J_{\ell}\right)\left\{\mathrm{d} P_{\ell}\right\} W\left[\left\{P_{\ell}\right\}\right]\right\} \\
\times & \frac{\prod_{\ell=1}^{k}\left\{\sum_{\sigma_{\ell}} \hat{P}_{\ell}\left(\sigma_{\ell} \mid J_{\ell} s\right)\right\} \delta\left[h-\sum_{\ell=1}^{k} J_{\ell} \sigma_{\ell}-\theta\right]}{Z\left[\left\{P_{1}, \ldots, P_{k}\right\}\right]}, \\
A\left[s, s^{\prime} ; h, h^{\prime} ; \tilde{s}\right] & =\sum_{k \geqslant 0} \frac{c^{k}}{k !} \mathrm{e}^{-c} \int \prod_{\ell=1}^{k}\left\{\mathrm{~d} J_{\ell} P\left(J_{\ell}\right)\left\{\mathrm{d} P_{\ell}\right\} W\left[\left\{P_{\ell}\right\}\right]\right\} \\
& \times \sum_{m \geqslant 0} \frac{c^{m}}{m !} \mathrm{e}^{-c} \int \prod_{r=1}^{m}\left\{\mathrm{~d} J_{r}^{\prime} P\left(J_{r}^{\prime}\right)\left\{\mathrm{d} Q_{r}\right\} W\left[\left\{Q_{r}\right\}\right]\right\}
\end{aligned}
$$




$$
\begin{aligned}
& \times\left\langle\prod_{\ell=1}^{k}\left\{\sum_{\sigma_{\ell}} \hat{P}_{\ell}\left(\sigma_{\ell} \mid J_{\ell} s^{\prime}\right)\right\} \delta\left[h^{\prime}-\sum_{\ell=1}^{k} J_{\ell} \sigma_{\ell}-\theta-J s\right] d\left(s^{\prime}, h^{\prime}\right)\right. \\
& \times \prod_{r=1}^{m}\left\{\sum_{\sigma_{r}} \hat{Q}_{r}\left(\sigma_{r} \mid J_{r}^{\prime} s\right)\right\} \delta\left[h-\sum_{r=1}^{m} J_{r}^{\prime} \sigma_{r}-\theta-J s^{\prime}+2 J \tilde{s}\right] d(s, h+2 J \tilde{S}) \\
& \times\left[\sum_{\sigma \sigma^{\prime}} \prod_{\ell=1}^{k}\left\{\sum_{\sigma_{\ell}} \hat{P}_{\ell}\left(\sigma_{\ell} \mid J_{\ell} \sigma\right)\right\} d\left(\sigma, \sum_{\ell=1}^{k} J_{\ell} \sigma_{\ell}+\theta+J \sigma^{\prime}\right)\right. \\
& \left.\left.\times \prod_{r=1}^{m}\left\{\sum_{\sigma_{r}} \hat{Q}_{r}\left(\sigma_{r} \mid J_{r}^{\prime} \sigma^{\prime}\right)\right\} d\left(\sigma^{\prime}, \sum_{r=1}^{m} J_{r}^{\prime} \sigma_{r}+\theta+J \sigma\right)\right]^{-1}\right\rangle_{J}
\end{aligned}
$$

Our theory requires the solution of the saddle-point equations (38) and (39) for the functional distribution $W[\{P\}]$ and the function $d(s, h)$. These are functional relations, and is generally not possible to solve them analytically. Furthermore, the imaginary arguments in (38) induce further complications in the numerical solution. To simplify matters we assume that for $x \in \mathbb{R}$ the Fourier transforms $\hat{P}(\sigma \mid x)$ are real-valued, and we define a corresponding functional distribution

$$
\tilde{W}[\{\hat{P}\}]=\int\{\mathrm{d} P\} W[\{P\}] \prod_{\sigma x} \delta\left[\hat{P}(\sigma \mid x)-\int \mathrm{d} \hat{h} P(\sigma, \hat{h}) \mathrm{e}^{-\mathrm{i} \hat{h} x}\right] .
$$

$\tilde{W}$ is normalized by construction, but the $\hat{P}(\sigma \mid x)$ need not be. We transform our problem into the language of $\tilde{W}$ by inserting (38) into (41) and integrating over $\{P\}$ :

$$
\begin{aligned}
\tilde{W}[\{\hat{P}\}]= & \sum_{k \geqslant 0} \frac{c^{k}}{k !} \mathrm{e}^{-c} \int \prod_{\ell=1}^{k}\left\{\mathrm{~d} J_{\ell} P\left(J_{\ell}\right)\left\{\mathrm{d} \hat{P}_{\ell}\right\} \tilde{W}\left[\left\{\hat{P}_{\ell}\right\}\right]\right\} \\
& \times \prod_{\sigma x} \delta\left[\hat{P}(\sigma \mid x)-\frac{\prod_{\ell=1}^{k}\left\{\sum_{\sigma_{\ell}} \hat{P}_{\ell}\left(\sigma_{\ell} \mid J_{\ell} \sigma\right)\right\} d\left(\sigma, \sum_{\ell=1}^{k} J_{\ell} \sigma_{\ell}+\theta+x\right)}{Z\left[\left\{\hat{P}_{1}, \ldots, \hat{P}_{k}\right\}\right]}\right],
\end{aligned}
$$

where $Z\left[\left\{\hat{P}_{1}, \ldots, \hat{P}_{k}\right\}\right]=\sum_{\sigma} \prod_{\ell=1}^{k}\left\{\sum_{\sigma_{\ell}} \hat{P}_{\ell}\left(\sigma_{\ell} \mid J_{\ell} \sigma\right)\right\} d\left(\sigma, \sum_{\ell=1}^{k} J_{\ell} \sigma_{\ell}+\theta\right)$.

Our previous results (39) and (40) under $W \rightarrow \tilde{W}$ take the form

$$
\begin{aligned}
D(s, h)=d(s, & h) \sum_{k \geqslant 0} \frac{c^{k}}{k !} \mathrm{e}^{-c} \int \prod_{\ell=1}^{k}\left\{\mathrm{~d} J_{\ell} P\left(J_{\ell}\right)\left\{\mathrm{d} \hat{P}_{\ell}\right\} \tilde{W}\left[\left\{\hat{P}_{\ell}\right\}\right]\right\} \\
& \times \frac{\prod_{\ell=1}^{k}\left\{\sum_{\sigma_{\ell}} \hat{P}_{\ell}\left(\sigma_{\ell} \mid J_{\ell} s\right)\right\} \delta\left[h-\sum_{\ell=1}^{k} J_{\ell} \sigma_{\ell}-\theta\right]}{Z\left[\left\{\hat{P}_{1}, \ldots, \hat{P}_{k}\right\}\right]}
\end{aligned}
$$

and

$$
\begin{aligned}
A\left[s, s^{\prime} ; h, h^{\prime} ; \tilde{s}\right] & =\sum_{k \geqslant 0} \frac{c^{k}}{k !} \mathrm{e}^{-c} \int \prod_{\ell=1}^{k}\left\{\mathrm{~d} J_{\ell} P\left(J_{\ell}\right)\left\{\mathrm{d} \hat{P}_{\ell}\right\} \tilde{W}\left[\left\{\hat{P}_{\ell}\right\}\right]\right\} \\
& \times \sum_{m \geqslant 0} \frac{c^{m}}{m !} \mathrm{e}^{-c} \int \prod_{r=1}^{m}\left\{\mathrm{~d} J_{r}^{\prime} d P\left(J_{r}^{\prime}\right)\left\{\mathrm{d} \hat{Q}_{r}\right\} \tilde{W}\left[\left\{\hat{Q}_{r}\right\}\right]\right\} \\
& \times\left\langle\prod_{\ell=1}^{k}\left\{\sum_{\sigma_{\ell}} \hat{P}_{\ell}\left(\sigma_{\ell} \mid J_{\ell} s^{\prime}\right)\right\} \delta\left[h^{\prime}-\sum_{\ell=1}^{k} J_{\ell} \sigma_{\ell}-\theta-J s\right] d\left(s^{\prime}, h^{\prime}\right)\right.
\end{aligned}
$$




$$
\begin{aligned}
& \times \prod_{r=1}^{m}\left\{\sum_{\sigma_{r}} \hat{Q}_{r}\left(\sigma_{r} \mid J_{r}^{\prime} s\right)\right\} \delta\left[h-\sum_{r=1}^{m} J_{r}^{\prime} \sigma_{r}-\theta-J s^{\prime}+2 J \tilde{s}\right] d(s, h+2 J \tilde{s}) \\
& \times\left[\sum_{\sigma \sigma^{\prime}} \prod_{\ell=1}^{k}\left\{\sum_{\sigma_{\ell}} \hat{P}_{\ell}\left(\sigma_{\ell} \mid J_{\ell} \sigma\right)\right\} d\left(\sigma, \sum_{\ell=1}^{k} J_{\ell} \sigma_{\ell}+\theta+J \sigma^{\prime}\right)\right. \\
& \left.\left.\times \prod_{r=1}^{m}\left\{\sum_{\sigma_{r}} \hat{Q}_{r}\left(\sigma_{r} \mid J_{r}^{\prime} \sigma^{\prime}\right)\right\} d\left(\sigma^{\prime}, \sum_{r=1}^{m} J_{r}^{\prime} \sigma_{r}+\theta+J \sigma\right)\right]^{-1}\right\rangle_{J}
\end{aligned}
$$

Equations (42)-(44) are the final analytic results within the replica-symmetry theory. They complement and close the diffusion equation (16). We may now proceed to the solution of (16) by iterating the following recipe from time $t=0$ onwards: at any time point $t$, we use the instantaneous distribution $D_{t}(s, h)$ to solve equations (42) and (43) numerically for $\tilde{W}[\{\hat{P}\}]$ and $d(s, h)$ via a population dynamics algorithm [6], the result of which is then used to compute kernel (44), and to iterate (16) over the next infinitesimal time step $t \rightarrow t+\mathrm{d} t$.

The numerical implementation of this theory is dependent on the nature of local fields (2). In the models with discrete fields (e.g. the FC Ising ferromagnet), the dynamical equation (16) reduces to the system of ordinary differential equations and the population dynamics can be implemented as in the vertex cover model (section 4) along the lines of appendix E. In a general case, where the fields can take any real value, one has to solve a PDE, by any standard method, and the population dynamics equations (42) and (43) can be solved, e.g. using the methods outlined in [25].

\section{Application of the theory to the vertex cover problem}

In this section, we show how the theory developed in the previous sections allows us to study analytically the Monte Carlo dynamics (extended with appropriate stochastic cooling schedules of the simulated annealing type) of the so-called minimum vertex cover optimization problem.

\subsection{The minimal vertex cover problem}

We start with the definitions. Let $G=(V, E)$ be a graph defined by a set of $N$ vertices $V=\{1, \ldots, N\}$ and a set of undirected edges $E=\{(i, j)\}$, where $i, j \in V$ and there is no distinction between $(i, j)$ and $(j, i)$. A vertex cover (VC) of a graph $G$ is a subset $V_{\mathrm{VC}} \subseteq V$ of vertices, such that for all edges $(i, j) \in E$ either $i \in V_{\mathrm{VC}}$ or $j \in V_{\mathrm{VC}}$ or both. The vertices in $V_{\mathrm{VC}}$ are called covered, and those in $V \backslash V_{\mathrm{VC}}$ uncovered. Similarly, an edge $(i, j)$ is said to be covered if at least one of the vertices $\{i, j\}$ is in $V_{\mathrm{VC}}$. The minimum vertex cover problem is the following optimization problem: find a vertex cover set $V_{\mathrm{VC}}$ of minimal cardinality, for a given graph $G$, and compute the fraction $x_{c}(G)=\left|V_{\mathrm{VC}}\right| / N$. The corresponding decision problem, to find whether for a given graph $G$ a VC of fixed cardinality $x=\left|V_{\mathrm{VC}}\right| / N$ exists, is known to belong to the class of NP-complete problems [26]; i.e., it is conjectured that no algorithm of polynomial time complexity in $N$ (or in the number of edges $M$ ) exists to solve it. All algorithms known to date indeed have exponential time complexity. The introduction of graph ensembles allows us to study typical instances of the minimal VC problem, and quantify the average properties. For instance, it was found that for large Poissonian random graphs (3) the fraction of covered vertices in a minimal vertex cover $x_{c}(G)$ depends on the average connectivity $c$ only, i.e. $x_{c}(G)=x_{c}(c)$. Rigorous lower and upper bounds for $x_{c}(c)$ were 
derived in [27]

$$
x_{l}(c)<x_{c}(c)<1-\ln (c) / c,
$$

where $x_{l}(c)$ is a solution of

$$
x \ln x+(1-x) \ln (1-x)-\frac{1}{2} c(1-x)^{2}=0 .
$$

The lower bound coincides with the annealed bound calculated within statistical mechanics, see, e.g. [28]. The asymptotic form of $x_{c}(c)$ for large $c$ was given in [29]

$$
x_{c}(c)=1-2[1+\ln (c)-\ln (\ln (c))-\ln (2)] / c+o\left(c^{-1}\right) .
$$

Since in VC problems a vertex is either covered or uncovered, one can map the VC problem in to an Ising model [10]: ${ }^{2}$ for any subset $U \subseteq V$ we define $\sigma_{i}=1$ if $i \in U$, and $\sigma_{i}=-1$ if $i \notin U$. We define a corresponding Hamiltonian for the state $\sigma=\left(\sigma_{1}, \ldots, \sigma_{N}\right)$ which simply counts the number of uncovered edges:

$$
H(\sigma)=\sum_{i<j} c_{i j} \delta_{\sigma_{i},-1} \delta_{\sigma_{j},-1}
$$

Solving the VC problem for a given relative cardinality $x$ then reduces to minimizing $H(\sigma)$ under the constraint $\sum_{i} \delta_{\sigma_{i}, 1}=x N$, which in terms of the Ising spins implies

$$
\frac{1}{N} \sum_{i} \sigma_{i}=2 x-1 .
$$

The study of VC has thereby been connected to the study of the ground states of an Ising spin system, within equilibrium statistical mechanics. This enabled the computation of the relative size of minimal VCs for typical large graph instances of FC ensembles, by averaging the free energy of the spin system over all graphs in the ensemble. Within the RS ansatz, this resulted for the Poissonian graphs in $[10,30]$

$$
x_{c}(c)=1-\frac{2 W(c)+W^{2}(c)}{2 c} .
$$

Here, $W(c)$ is the Lambert $W$-function [31], defined as the real solution of $c=W \mathrm{e}^{W}$. This result implies that almost all graphs are coverable with $x N$ vertices for $x>x_{c}(c)$ and not coverable for $x<x_{c}(c)$. It was proved to be exact for $c \leqslant \exp (1)$ [32], but for $c>\exp (1)$ equation (50) underestimates the empirical values of $x_{c}(c)$ obtained by numeric simulation [10], and for $c \geqslant 20.7$ it even violates the lower bound (46). The explanation was found to be that at $c=\exp (1)$ the assumed RS breaks down [10, 30], and replica-symmetry breaking (RSB) occurs. The one-step RSB solution was computed in [33] via the cavity method; its agreement with the numeric results [10] improves upon the RS calculation (50) and approaches (47) correctly for large $c$, yet the one-step RSB solution is still incorrect for $c>\exp (1)$ [33]. A more recent result obtained in [34] is in good agreement with both the numerical simulations [10] for $c \leqslant 10.0$, and with the asymptotic form (47) for large $c$. A few and limited analytic studies of the dynamics of algorithmic solutions of the VC problem were carried out only for a simple backtracking algorithm [35], and for more complex heuristic [36, 37] algorithms. In this paper, in contrast, we consider a more physical dynamics, inspired by the connection with a ground-state search in the Ising spin systems.

2 An alternative representation involves mapping the VC problem into a hard-sphere lattice gas [30]. 


\subsection{Dynamic replica analysis of the vertex cover problem}

In this section, we analyse the Monte Carlo dynamics for the minimum VC problem of type (5), where we allow the temperature $T$ to vary over time such that $\lim _{t \rightarrow \infty} T(t)=0$, but sufficiently slowly so that in (5) we may simply substitute $\beta \rightarrow \beta(t)$. We map the VC problem into the Ising model (48), and impose the constraint (49) in a 'soft' way, by adding an extra term to the Hamiltonian (48)

$$
\tilde{H}(\boldsymbol{\sigma})=\sum_{i<j} c_{i j} \delta_{\sigma_{i},-1} \delta_{\sigma_{j},-1}-\lambda \sum_{i} \delta_{\sigma_{i},-1}
$$

(where $\lambda>0$ ), which ensures that among the states $\sigma$ that minimize (51), those with the smallest sum $\sum_{i} \delta_{\sigma_{i}, 1}$ (i.e. minimal cover) are preferred. The Glauber dynamics associated with the Hamiltonian (51) is indeed of type (1), with the local field

$$
h_{i}(\sigma)=J \sum_{j \neq i} c_{i j} \delta_{\sigma_{j},-1}+\theta
$$

where $J=\frac{1}{2}$ and $\theta=-\frac{1}{2} \lambda$. The fields could also have been written in the more standard form $h_{i}(\boldsymbol{\sigma}) \stackrel{2}{=} \sum_{j} J_{i j} \sigma_{j}+\theta_{i}$, but this would have required the site-dependent random $\theta_{i}$ which involve the connectivity variables $c_{i j}$. The consequences for our theory of changing the local fields from the conventional form (2) to (52) are minor. The diffusion equation (16) remains unchanged; the only difference is in definition of distribution (11), which now becomes

$$
D(s, h ; \boldsymbol{\sigma})=\frac{1}{N} \sum_{i} \delta_{s, \sigma_{i}} \delta\left[h-J \sum_{j \neq i} c_{i j} \delta_{\sigma_{j},-1}-\theta\right]
$$

and kernel (15), which changes to

$$
\tilde{A}\left[s, s^{\prime} ; h, h^{\prime} ; \tilde{s}\right]=\left\langle\frac{1}{c N} \sum_{i j} \delta_{s^{\prime}, \sigma_{i}} \delta_{s, \sigma_{j}} c_{i j} \delta\left[h^{\prime}-h_{i}(\sigma)\right] \delta\left[h-h_{j}(\sigma)-J \tilde{s}\right]\right\rangle_{D ; t},
$$

where $\tilde{s} \in\left\{0, s^{\prime}\right\}$ and $h_{i}(\sigma)$ is given by (52). Next, we compute the consequences of defining (52) within the replica calculations. This involves only minor alterations of the steps taken in section 3 , and we readily obtain the new expression that replaces our previous (28) (where in $\mathrm{VC}$ there is of course no longer a need to average over $J$ ):

$$
A\left[s, s^{\prime} ; h, h^{\prime} ; \tilde{s}\right]=\lim _{n \rightarrow 0} \frac{\left\langle\delta_{s^{\prime}, \sigma_{1}} \delta_{s, \sigma_{1}^{\prime}} \delta\left[h^{\prime}-H_{1}\right] \delta\left[h-H_{1}^{\prime}-J \tilde{s}\right] \mathrm{e}^{-\mathrm{i} J \sum_{\alpha}\left[\hat{h}_{\alpha} \delta_{\sigma_{\alpha}^{\prime},-1}+\hat{h}_{\alpha}^{\prime} \delta_{\sigma_{\alpha},-1}\right]}\right\rangle_{M, M^{\prime}}}{\left\langle\mathrm{e}^{-\mathrm{i} J \sum_{\alpha}\left[\hat{h}_{\alpha} \delta_{\sigma_{\alpha}^{\prime},-1}+\hat{h}_{\alpha}^{\prime} \delta_{\sigma_{\alpha},-1}\right]}\right\rangle_{M, M^{\prime}}} .
$$

The saddle-point equations (29), (30) remain unaltered, with the $\langle\ldots\rangle_{M}$ averages given by equation (25), but now the associated measure takes the new form:

$$
\begin{aligned}
M[\boldsymbol{H}, \hat{\boldsymbol{h}}, \boldsymbol{\sigma} \mid \theta] & =\exp \left\{\mathrm{i} \hat{\boldsymbol{h}} \cdot[\boldsymbol{H}-\boldsymbol{\theta}]-\mathrm{i} \sum_{\alpha} \hat{D}_{\alpha}\left(\sigma_{\alpha}, H_{\alpha}\right)\right. \\
& \left.+c \sum_{\sigma^{\prime}} \int \mathrm{d} \hat{\boldsymbol{h}}^{\prime} P\left(\boldsymbol{\sigma}^{\prime}, \hat{\boldsymbol{h}}^{\prime}\right)\left[\mathrm{e}^{-\mathrm{i} J \sum_{\alpha}\left[\hat{h}_{\alpha} \delta_{\sigma_{\alpha}^{\prime},-1}+\hat{h}_{\alpha}^{\prime} \delta_{\sigma_{\alpha},-1}\right]}-1\right]\right\} .
\end{aligned}
$$

The only changes to the earlier theory that are induced by the introduction of (52) are in the imaginary arguments of the exponential function in (55) and (56). We can therefore 
derive the RS version of the theory for VC dynamics simply by replacing $\sigma_{\alpha} \rightarrow \delta_{\sigma_{\alpha},-1}$ and $P\left(J_{\ell}\right) \rightarrow \delta\left(J_{\ell}-J\right)$ in equations (38)-(40) of section 3.2. This results in

$W[\{P\}]=\sum_{k \geqslant 0} \frac{c^{k}}{k !} \mathrm{e}^{-c} \int \prod_{\ell=1}^{k}\left\{\left\{\mathrm{~d} P_{\ell}\right\} W\left[\left\{P_{l}\right\}\right]\right\}$

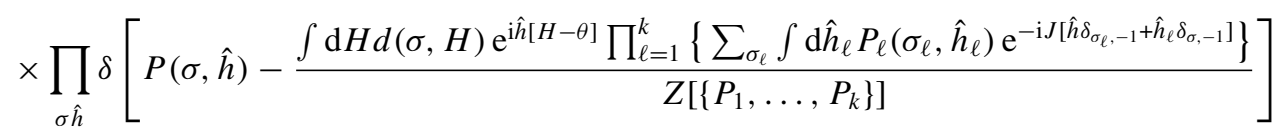

and, with the Fourier transforms $\hat{P}(\sigma \mid x)=\int \mathrm{d} \hat{h} P(\sigma, \hat{h}) \mathrm{e}^{-\mathrm{i} \hat{h} x}$,

$$
\begin{aligned}
D(s, h)=d(s, & h) \sum_{k \geqslant 0} \frac{c^{k}}{k !} \mathrm{e}^{-c} \int \prod_{\ell=1}^{k}\left\{\left\{\mathrm{~d} P_{\ell}\right\} W\left[\left\{P_{\ell}\right\}\right]\right\} \\
& \times \frac{\prod_{\ell=1}^{k}\left\{\sum_{\sigma_{\ell}} \hat{P}_{\ell}\left(\sigma_{\ell} \mid J \delta_{s,-1}\right)\right\} \delta\left[h-J \sum_{\ell=1}^{k} \delta_{\sigma_{\ell},-1}-\theta\right]}{Z\left[\left\{P_{1}, \ldots, P_{k}\right\}\right]}, \\
A\left[s, s^{\prime} ; h, h^{\prime} ; \tilde{s}\right] & =\sum_{k \geqslant 0} \frac{c^{k}}{k !} \mathrm{e}^{-c} \int \prod_{\ell=1}^{k}\left\{\left\{\mathrm{~d} P_{\ell}\right\} W\left[\left\{P_{\ell}\right\}\right] \sum_{\sigma_{\ell}} \hat{P}_{\ell}\left(\sigma_{\ell} \mid J \delta_{s^{\prime},-1}\right)\right\} \\
& \times \sum_{m \geqslant 0} \frac{c^{m}}{m !} \mathrm{e}^{-c} \int \prod_{r=1}^{m}\left\{\left\{\mathrm{~d} Q_{r}\right\} W\left[\left\{Q_{r}\right\}\right] \sum_{\sigma_{r}} \hat{Q}_{r}\left(\sigma_{r} \mid J \delta_{s,-1}\right)\right\} \\
& \times \delta\left[h^{\prime}-J \sum_{\ell=1}^{k} \delta_{\sigma_{\ell},-1}-\theta-J \delta_{s,-1}\right] d\left(s^{\prime}, h^{\prime}\right) \\
& \times \delta\left[h-J \sum_{r=1}^{m} \delta_{\sigma_{r},-1}-\theta-J \delta_{s^{\prime},-1}-J \tilde{s}\right] d(s, h-J \tilde{s}) \\
& \times\left[\sum_{\sigma \sigma^{\prime}} \prod_{\ell=1}^{k}\left\{\sum_{\sigma_{\ell}} \hat{P}_{\ell}\left(\sigma_{\ell} \mid J \delta_{\sigma,-1}\right)\right\} d\left(\sigma, J \sum_{\ell=1}^{k} \delta_{\sigma_{\ell},-1}+\theta+J \delta_{\sigma^{\prime},-1}\right)\right. \\
& \left.\times \prod_{r=1}^{m}\left\{\sum_{\sigma_{r}} \hat{Q}_{r}\left(\sigma_{r} \mid J \delta_{\sigma^{\prime},-1}\right)\right\} d\left(\sigma^{\prime}, J \sum_{r=1}^{m} \delta_{\sigma_{r},-1}+\theta+J \delta_{\sigma,-1}\right)\right]^{-1}
\end{aligned}
$$

As before we may switch to a measure defined directly on the relevant Fourier transforms, which in the case of $\mathrm{VC}$ simplifies further due to the uniform bonds $J$ :

$\tilde{W}[\{\hat{P}\}]=\int\{\mathrm{d} P\} W[\{P\}] \prod_{\sigma \sigma^{\prime}} \delta\left[\hat{P}\left(\sigma \mid J \delta_{\sigma^{\prime},-1}\right)-\int \mathrm{d} \hat{h} P(\sigma, \hat{h}) \mathrm{e}^{-\mathrm{i} \hat{h} J \delta_{\sigma^{\prime},-1}}\right]$.

Our RS equations now acquire the following form:

$$
\begin{aligned}
& \tilde{W}[\{\hat{P}\}]=\sum_{k \geqslant 0} \frac{c^{k}}{k !} \mathrm{e}^{-c} \int \prod_{\ell=1}^{k}\left\{\left\{\mathrm{~d} \hat{P}_{\ell}\right\} \tilde{W}\left[\left\{\hat{P}_{\ell}\right\}\right]\right\} \\
& \times \prod_{\sigma \sigma^{\prime}} \delta\left[\hat{P}\left(\sigma \mid J \delta_{\sigma^{\prime},-1}\right)-\frac{\prod_{\ell=1}^{k}\left\{\sum_{\sigma_{\ell}} \hat{P}_{\ell}\left(\sigma_{\ell} \mid J \delta_{\sigma,-1}\right)\right\} d\left(\sigma, J \sum_{\ell=1}^{k} \delta_{\sigma_{\ell},-1}+\theta+J \delta_{\sigma^{\prime},-1}\right)}{Z\left[\left\{\hat{P}_{1}, \ldots, \hat{P}_{k}\right\}\right]}\right]
\end{aligned}
$$




$$
\begin{aligned}
D(s, h)=d(s, h) \sum_{k \geqslant 0} \frac{c^{k}}{k !} \mathrm{e}^{-c} \int \prod_{\ell=1}^{k}\left\{\left\{\mathrm{~d} \hat{P}_{\ell}\right\} \tilde{W}\left[\left\{\hat{P}_{\ell}\right\}\right]\right\} \\
\times \frac{\prod_{\ell=1}^{k}\left\{\sum_{\sigma_{\ell}} \hat{P}_{\ell}\left(\sigma_{\ell} \mid J \delta_{s,-1}\right)\right\} \delta\left[h-J \sum_{\ell=1}^{k} \delta_{\sigma_{\ell},-1}-\theta\right]}{Z\left[\left\{\hat{P}_{1}, \ldots, \hat{P}_{k}\right\}\right]},
\end{aligned}
$$

where $Z\left[\left\{\hat{P}_{1}, \ldots, \hat{P}_{k}\right\}\right]=\sum_{\sigma} \prod_{\ell=1}^{k}\left\{\sum_{\sigma_{\ell}} \hat{P}_{\ell}\left(\sigma_{\ell} \mid J \delta_{\sigma,-1}\right)\right\} d\left(\sigma, J \sum_{\ell=1}^{k} \delta_{\sigma_{\ell},-1}+\theta\right)$, and

$$
\begin{aligned}
A\left[s, s^{\prime} ; h, h^{\prime} ; \tilde{s}\right] & =\sum_{k \geqslant 0} \frac{c^{k}}{k !} \mathrm{e}^{-c} \int \prod_{\ell=1}^{k}\left\{\left\{\mathrm{~d} \hat{P}_{\ell}\right\} \tilde{W}\left[\left\{\hat{P}_{\ell}\right\}\right] \sum_{\sigma_{\ell}} \hat{P}_{\ell}\left(\sigma_{\ell} \mid J \delta_{s^{\prime},-1}\right)\right\} \\
& \times \sum_{m \geqslant 0} \frac{c^{m}}{m !} \mathrm{e}^{-c} \int \prod_{r=1}^{m}\left\{\left\{\mathrm{~d} \hat{Q}_{r}\right\} \tilde{W}\left[\left\{\hat{Q}_{r}\right\}\right] \sum_{\sigma_{r}} \hat{Q}_{r}\left(\sigma_{r} \mid J \delta_{s,-1}\right)\right\} \\
& \times \delta\left[h^{\prime}-J \sum_{\ell=1}^{k} \delta_{\sigma_{\ell},-1}-\theta-J \delta_{s,-1}\right] d\left(s^{\prime}, h^{\prime}\right) \\
& \times \delta\left[h-J \sum_{r=1}^{m} \delta_{\sigma_{r},-1}-\theta-J \delta_{s^{\prime},-1}-J \tilde{s}\right] d(s, h-J \tilde{s}) \\
& \times\left[\sum_{\sigma \sigma^{\prime}} \prod_{\ell=1}^{k}\left\{\sum_{\sigma_{\ell}} \hat{P}_{\ell}\left(\sigma_{\ell} \mid J \delta_{\sigma,-1}\right)\right\} d\left(\sigma, J \sum_{\ell=1}^{k} \delta_{\sigma_{\ell},-1}+\theta+J \delta_{\sigma^{\prime},-1}\right)\right. \\
& \left.\times \prod_{r=1}^{m}\left\{\sum_{\sigma_{r}} \hat{Q}_{r}\left(\sigma_{r} \mid J \delta_{\sigma^{\prime},-1}\right)\right\} d\left(\sigma^{\prime}, J \sum_{r=1}^{m} \delta_{\sigma_{r},-1}+\theta+J \delta_{\sigma,-1}\right)\right]^{-1} .
\end{aligned}
$$

Compared to the more general expression (42), in the VC case (61) the dimensionality of our problem has been reduced drastically, as $\tilde{W}$ is now a functional on the space of $2 \times 2$ matrices $\hat{P}\left(\sigma \mid J \delta_{\sigma^{\prime},-1}\right)$. Furthermore, the solutions of (61), (63) are of the following form, which is expected on physical grounds (given the non-random bonds $J$ in VC):

$$
\begin{aligned}
& D(s, h)=\sum_{n \geqslant 0} P(s, n) \delta(h-J n-\theta), \\
& \begin{aligned}
A\left[s, s^{\prime} ; h, h^{\prime} ; \tilde{s}\right] & =\sum_{n, n^{\prime} \geqslant 0} A\left[s, s^{\prime} ; n, n^{\prime}\right] \delta\left[h^{\prime}-J n^{\prime}-\theta-J \delta_{s,-1}\right] \\
& \times \delta\left[h-J n-\theta-J \delta_{s^{\prime},-1}-J \tilde{s}\right],
\end{aligned}
\end{aligned}
$$

where $P(s, n)$ and $A\left[s, s^{\prime} ; n, n^{\prime}\right]$ (with $s, s^{\prime} \in\{-1,1\}$ and $\left.n, n^{\prime} \in\{0,1,2, \ldots\}\right)$ are solved from

$$
\begin{aligned}
P(s, n)=\sum_{k \geqslant 0} \frac{c^{k}}{k !} \mathrm{e}^{-c} \int \prod_{\ell=1}^{k}\left\{\left\{\mathrm{~d} \hat{P}_{\ell}\right\} \tilde{W}\left[\left\{\hat{P}_{\ell}\right\}\right]\right\} \\
\times \frac{\prod_{\ell=1}^{k}\left\{\sum_{\sigma_{\ell}} \hat{P}_{\ell}\left(\sigma_{\ell} \mid J \delta_{s,-1}\right)\right\} d(s, J n+\theta) \delta_{n, \sum_{\ell=1}^{k} \delta_{\sigma_{\ell},-1}}}{\sum_{\sigma} \prod_{\ell=1}^{k}\left\{\sum_{\sigma_{\ell}} \hat{P}_{\ell}\left(\sigma_{\ell} \mid J \delta_{\sigma,-1}\right)\right\} d\left(\sigma, J \sum_{\ell=1}^{k} \delta_{\sigma_{\ell},-1}+\theta\right)}
\end{aligned}
$$

and

$$
A\left[s, s^{\prime} ; n, n^{\prime}\right]=\sum_{k \geqslant 0} \frac{c^{k}}{k !} \mathrm{e}^{-c} \int \prod_{\ell=1}^{k}\left\{\left\{\mathrm{~d} \hat{P}_{\ell}\right\} \tilde{W}\left[\left\{\hat{P}_{\ell}\right\}\right] \sum_{\sigma_{\ell}} \hat{P}_{\ell}\left(\sigma_{\ell} \mid J \delta_{s^{\prime},-1}\right)\right\}
$$




$$
\begin{aligned}
& \times \sum_{m \geqslant 0} \frac{c^{m}}{m !} \mathrm{e}^{-c} \int \prod_{r=1}^{m}\left\{\left\{\mathrm{~d} \hat{Q}_{r}\right\} \tilde{W}\left[\left\{\hat{Q}_{r}\right\}\right] \sum_{\sigma_{r}} \hat{Q}_{r}\left(\sigma_{r} \mid J \delta_{s,-1}\right)\right\} \\
& \times d\left(s^{\prime}, J n^{\prime}+\theta+J \delta_{s,-1}\right) \delta_{n^{\prime}, \sum_{\ell=1}^{k} \delta_{\sigma_{\ell},-1}} \\
& \times d\left(s, J n+\theta+J \delta_{s^{\prime},-1}\right) \delta_{n, \sum_{r=1}^{m} \delta_{\sigma_{r},-1}} \\
& \times\left[\sum_{\sigma \sigma^{\prime}} \prod_{\ell=1}^{k}\left[\sum_{\sigma_{\ell}} \hat{P}_{\ell}\left(\sigma_{\ell} \mid J \delta_{\sigma,-1}\right)\right] d\left(\sigma, J \sum_{\ell=1}^{k} \delta_{\sigma_{\ell},-1}+\theta+J \delta_{\sigma^{\prime},-1}\right)\right. \\
& \left.\times \prod_{r=1}^{m}\left[\sum_{\sigma_{r}} \hat{Q}_{r}\left(\sigma_{r} \mid J \delta_{\sigma^{\prime},-1}\right)\right] d\left(\sigma^{\prime}, J \sum_{r=1}^{m} \delta_{\sigma_{r},-1}+\theta+J \delta_{\sigma,-1}\right)\right]^{-1} .
\end{aligned}
$$

The simplified form of kernels (64), (65) subsequently allows us to transform the main dynamical equation (16), which is a PDE, into the following system of ordinary differential equations (see appendix $\mathrm{C}$ for details):

$$
\begin{aligned}
\frac{\mathrm{d}}{\mathrm{d} t} P(s, 0)= & \frac{1}{2}[1+s \tanh [\beta \theta]] P(-s, 0)-\frac{1}{2}[1-s \tanh [\beta \theta]] P(s, 0) \\
& +\frac{1}{2} c \sum_{n^{\prime} \geqslant 0}\left[1+\tanh \left[\beta J n^{\prime}+\beta \theta+\beta J \delta_{s,-1}\right]\right] A\left[s,-1 ; 0, n^{\prime}\right] \\
& -\frac{1}{2} c \sum_{n^{\prime} \geqslant 0}\left[1-\tanh \left[\beta J n^{\prime}+\beta \theta+\beta J \delta_{s,-1}\right]\right] A\left[s, 1 ; 0, n^{\prime}\right],
\end{aligned}
$$

whereas for $n>0$ we have

$$
\begin{aligned}
\frac{\mathrm{d}}{\mathrm{d} t} P(s, n)= & \frac{1}{2}[1+s \tanh [\beta J n+\beta \theta]] P(-s, n)-\frac{1}{2}[1-s \tanh [\beta J n+\beta \theta]] P(s, n) \\
& +\frac{1}{2} c \sum_{n^{\prime} \geqslant 0}\left[1-\tanh \left[\beta J n^{\prime}+\beta \theta+\beta J \delta_{s,-1}\right]\right] A\left[s, 1 ; n-1, n^{\prime}\right] \\
& +\frac{1}{2} c \sum_{n^{\prime} \geqslant 0}\left[1+\tanh \left[\beta J n^{\prime}+\beta \theta+\beta J \delta_{s,-1}\right]\right] A\left[s,-1 ; n, n^{\prime}\right] \\
& -\frac{1}{2} c \sum_{n^{\prime} \geqslant 0}\left[1-\tanh \left[\beta J n^{\prime}+\beta \theta+\beta J \delta_{s,-1}\right]\right] A\left[s, 1 ; n, n^{\prime}\right] \\
& -\frac{1}{2} c \sum_{n^{\prime} \geqslant 0}\left[1+\tanh \left[\beta J n^{\prime}+\beta \theta+\beta J \delta_{s,-1}\right]\right] A\left[s,-1 ; n-1, n^{\prime}\right] .
\end{aligned}
$$

Equations (61) and (66)-(69) are the final results of our dynamical replica analysis. They can be solved numerically, using population dynamics for the functional saddle-point equations (see appendix E for details) and any standard method for the system of ordinary differential equations. If we allow for temperature adaptation, namely $\beta \rightarrow \beta(t)$, and restrict ourselves to those cooling protocols where $\beta(t)$ changes only on $\mathcal{O}\left(N^{0}\right)$ time scales, we may simply make the replacement $\beta \rightarrow \beta(t)$ in the above equations.

\section{Tests of the VC theory against numerical simulations}

To test our theoretic predictions for the evolution of observables in the Glauber algorithm with stochastic cooling running on the VC problem, we compare the results of solving numerically 
the system of dynamical equations (69) with the results of numerical simulations. We solve (69) using a simple first-order Euler method, i.e. we iterate the iteration

$$
P_{\ell+1}(s, n)=P_{\ell}(s, n)+h \Gamma\left[s, n ; P_{\ell}(\ldots) ; A_{\ell}[\ldots]\right], \quad t_{\ell}=\ell h,
$$

where $n \in\{0,1, \ldots, L(c)\}$ and $\Gamma[\ldots]$ is a short-hand for the right-hand side of (68), (69). Here, $L(c)$ denotes a suitable cut-off value that increases monotonically with the average connectivity $c$ in (3), and $0<h \ll 1$. At each discrete time-step $\ell$ of this iteration, we solve the RS equations (61), (66) via a population dynamics algorithm (see appendix E) and compute kernel (67). Solving (69) requires a significant numerical effort, the bulk of which is devoted to solving equations (61), (66), where the computation of a typical object (E.1) requires typically $O\left(2^{c}\right)$ basic operations. Although we expect that for sufficiently small $h$ the changes in the statistical properties of the population between consecutive iterative time steps in (70) are small, the fact that the running time of the algorithm (70) grows exponentially with $c$ restricts the scope of simulation experiments. For each choice of control parameters, our experimental protocol has been the following. First, we generate a large random Poissonian graph with the required connectivity $c$. Then we run the algorithm (1) with the local fields (52), from an initial spin configuration where the individual spins are drawn randomly and independently from distribution (D.1). We then let the system evolve according to the Glauber algorithm, but with the temperature $T(t)$ decreasing in stages (to achieve stochastic cooling), while we record the evolution of two macroscopic-order parameters, being the fraction $x$ of covered vertices

$$
x(\boldsymbol{\sigma})=\frac{1}{N} \sum_{i} \delta_{\sigma_{i}, 1}
$$

and the energy density $E$, which is proportional to the fraction of uncovered edges,

$$
E(\boldsymbol{\sigma})=\frac{1}{N} \sum_{i<j} c_{i j} \delta_{\sigma_{i},-1} \delta_{\sigma_{j},-1} .
$$

For a state $\sigma$ to represent an acceptable vertex cover, it must have $E(\sigma)=0$. For such a cover to be minimal, we want in addition $x(\sigma)$ to be as small as possible.

In simulated annealing one starts a Monte Carlo dynamics at a high temperature $T(0)$, and then lowers it slowly in stages, allowing the system to equilibrate effectively along the way. The objective is for the algorithm not to get stuck in states that are only locally but not globally optimal. Determining the best cooling schedule $T(t)$ for achieving this, however, is highly non-trivial; furthermore, equilibration times in VC-type optimization problems can scale exponentially in the system size. Here we did not attempt to optimize the cooling protocol but focused on the VC dynamics for simple stepwise temperature reductions. Our numerical simulations were carried out on random graphs with $N=10000$ vertices, with average connectivities $c \in\{0.5,1,1.5,2,2.5,3,3.5\}$. The values of the parameters $J=1, \theta=-0.99$, the initial covered fraction $x_{0}=N^{-1} \sum_{i} \delta_{\sigma_{i}(0), 1}=0.9$ and the temperatures $T \in\{2,1,0.5,0\}$ (reduced in steps) were identical in all simulations. The initial conditions for (69) and the population dynamics were computed via equations (D.3)-(D.2) of appendix D. The value of the step size in (70) was $h=0.1$ and the cut-off values $L(c)$ for $n$ were chosen according to

\begin{tabular}{l|c|c|c|c|c|c|c}
$c$ & 0.5 & 1.0 & 1.5 & 2.0 & 2.5 & 3.0 & 3.5 \\
\hline$L(c)$ & 5 & 8 & 8 & 8 & 9 & 10 & 10
\end{tabular}

The size of the population was $\mathcal{N}=10000$ and the number of iterations typically needed for the population dynamics to converge was of order $10 \mathcal{N}$.

In figures 1-4, we compare the data obtained in our numerical simulations for $c \in$ $\{0.5,1,2,3\}$ with the results of solving (69) numerically. We observe that the overall agreement 

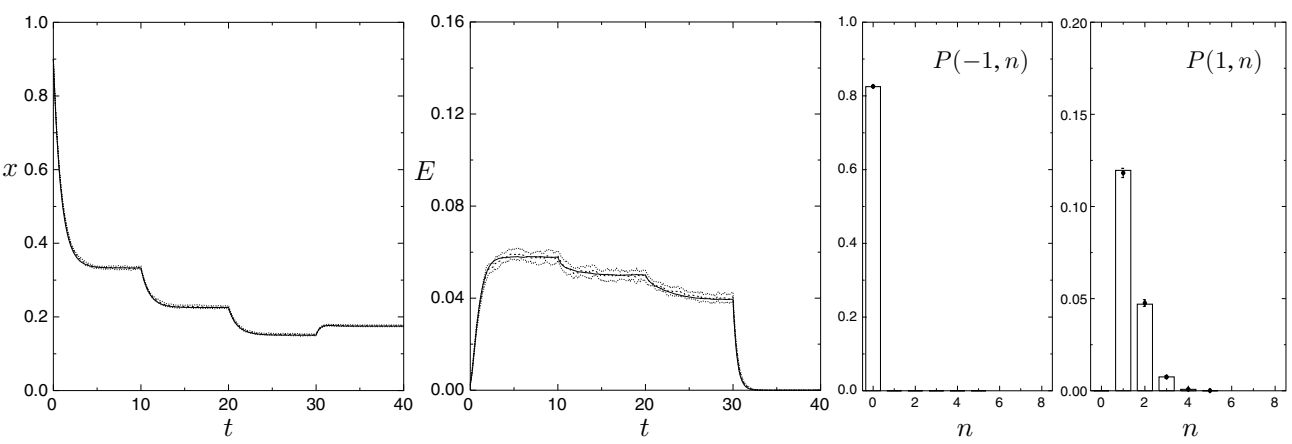

Figure 1. Left and middle: evolution of the fraction $x$ and the energy density $E$ in the VC algorithm with simulated annealing, for $c=0.5, J=1.0$ and $\theta=-0.99$. Time is measured in iterations per spin. Solid lines: RS theory. Dashed and dotted lines: average and average plus/minus standard deviation as measured over 100 simulation runs in systems with $N=10^{4}$ spins. The annealing schedule had four stages: (i) $T=2$ for $t \in[0,10]$, (ii) $T=1$ for $t \in[10,20]$, (iii) $T=0.5$ for $t \in[20,30]$ and (iv) $T=0$ for $t \in[30,40]$. Right: histograms (RS theory) of the two field distributions $P( \pm 1, n)$ at $t=40$, together with the corresponding simulation measurements (markers with error bars).
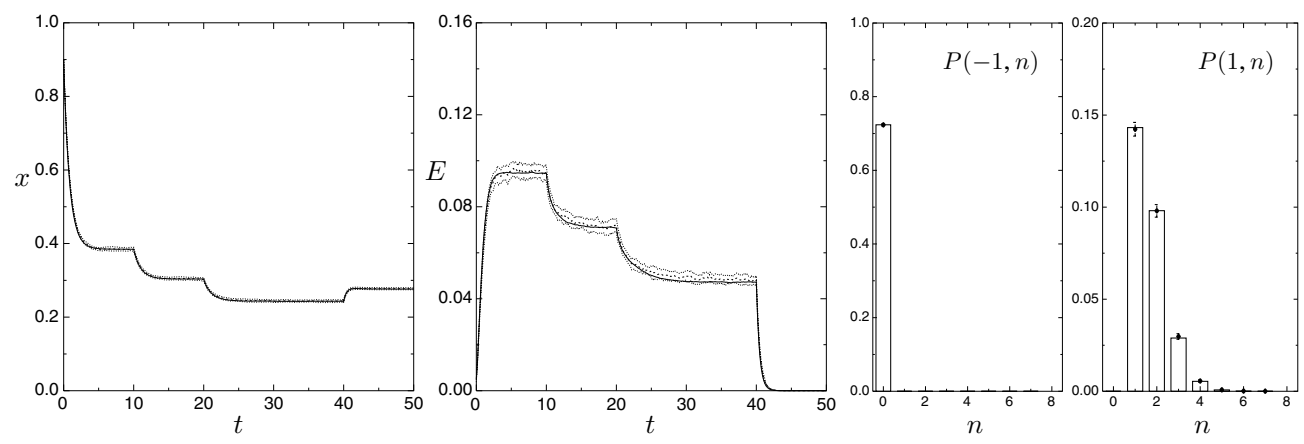

Figure 2. Left and middle: evolution of the fraction $x$ and the energy density $E$ in the VC algorithm with simulated annealing, for $c=1, J=1.0$ and $\theta=-0.99$. Time is measured in iterations per spin. Solid lines: RS theory. Dashed and dotted lines: average and average plus/minus standard deviation as measured over 100 simulation runs in systems with $N=10^{4}$ spins. The annealing schedule had four stages: (i) $T=2$ for $t \in[0,10]$, (ii) $T=1$ for $t \in[10,20]$, (iii) $T=0.5$ for $t \in[20,40]$ and (iv) $T=0$ for $t \in[40,50]$. Right: histograms (RS theory) of the two field distributions $P( \pm 1, n)$ at $t=50$, together with the corresponding simulation measurements (markers with error bars).

between theory and simulations is excellent. The RS theory also predicts correctly the joint spin-field statistics $P_{t}(s, n)$, see the right panels in figures 1-4; note the different vertical scales. The $P_{t}(-1, n)$ histograms, in figures $1-4$, show that all neighbours of uncovered vertices are covered, thus the configurations sampled at final times are indeed vertex covers. The deviations between theory and simulations are (as usual in DRT) confined to intermediate times, and limited to low temperatures in combination with high average connectivity, as shown in the insets of figures 3 and 4; but even there they remain within the error bars of the simulation data. Finally, in figure 5, we compare our results for the fraction of covered vertices $x$ as measured at termination of the algorithm with result (50) of equilibrium statistical 

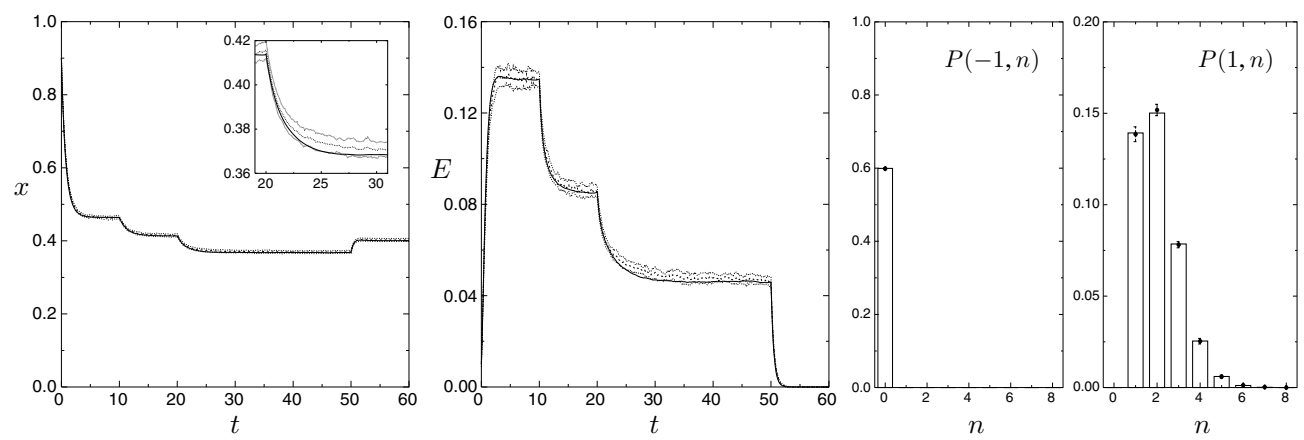

Figure 3. Left and middle: evolution of the fraction $x$ and the energy density $E$ in the VC algorithm with simulated annealing, for $c=2, J=1.0$ and $\theta=-0.99$. Time is measured in iterations per spin. Solid lines: RS theory. Dashed and dotted lines: average and average plus/minus standard deviation as measured over 100 simulation runs in systems with $N=10^{4}$ spins. The annealing schedule had four stages: (i) $T=2$ for $t \in[0,10]$, (ii) $T=1$ for $t \in[10,20]$, (iii) $T=0.5$ for $t \in[20,50]$ and (iv) $T=0$ for $t \in[50,60]$. The inset in the left figure shows an enlargement of the region $t \in[20,30]$, where the largest deviation between theory and simulation for $x$ is observed. Right: histograms (RS theory) of the two field distributions $P( \pm 1, n)$ at $t=60$, together with the corresponding simulation measurements (markers with error bars).
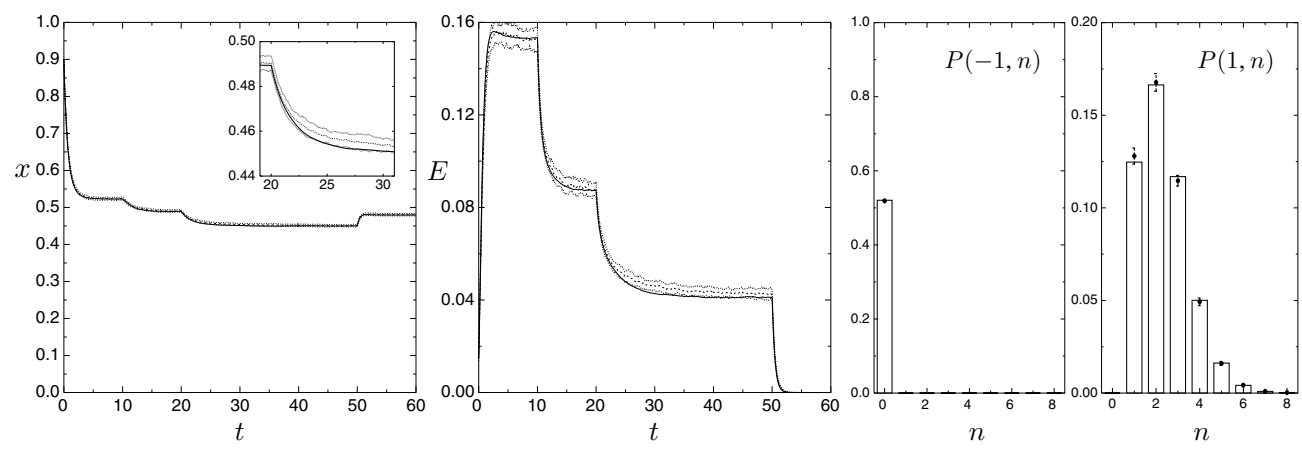

Figure 4. Left and middle: evolution of the fraction $x$ and the energy density $E$ in the VC algorithm with simulated annealing, for $c=3, J=1.0$ and $\theta=-0.99$. Time is measured in iterations per spin. Solid lines: RS theory. Dashed and dotted lines: average and average plus/minus standard deviation as measured over 100 simulation runs in systems with $N=10^{4}$ spins. The annealing schedule had four stages: (i) $T=2$ for $t \in[0,10]$, (ii) $T=1$ for $t \in[10,20]$, (iii) $T=0.5$ for $t \in[20,50]$ and (iv) $T=0$ for $t \in[50,60]$. The inset in the left figure shows an enlargement of the region $t \in[20,30]$, where the largest deviation between theory and simulation for $x$ is observed. Right: histograms (RS theory) of the two field distributions $P( \pm 1, n)$ at $t=60$, together with the corresponding simulation measurements (markers with error bars).

mechanics, as obtained (within the replica-symmetry ansatz) in [10]. Our data (markers, with virtually no difference between the observed values in simulations and the prediction of our dynamical theory) are seen to be close to the equilibrium prediction (50) (solid curve), but they overestimate slightly the size of the minimal vertex covers. This type of behaviour is not unusual in simulations, and suggests that more sophisticated annealing schemes must be used to achieve equilibration. Slightly more unexpected is the fact that our RS theory exhibits a similar overestimation of $x$. This, in combination with the fact that the static RS equations 


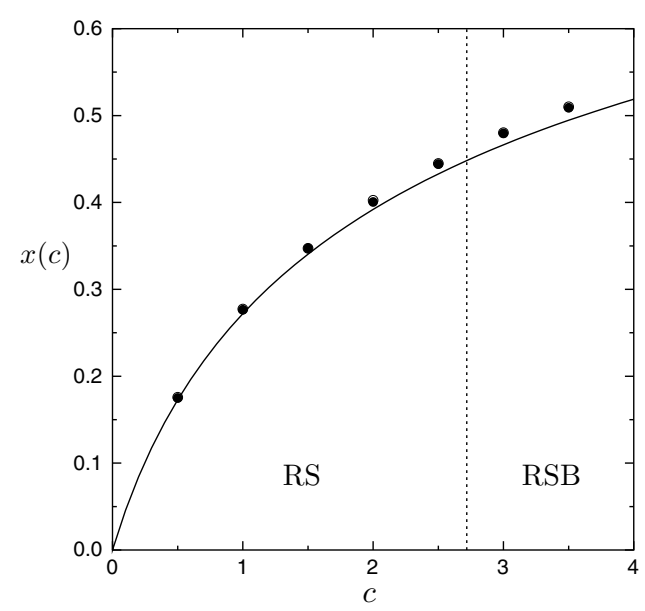

Figure 5. The fraction $x(c)$ of covered vertices in a minimal vertex cover as a function of the average connectivity $c$. Solid line: prediction of a static replica-symmetric calculation (exact for $c<e$; the value $c=e$ is shown as a vertical dashed line), as obtained in [10]. Symbols: the predicted final fraction $x$ of covered vertices in the vertex cover obtained from the present dynamics (when we have arrived at $T=0$ ), according to the RS dynamical replica method (which agrees perfectly with the simulations).

of [10] can be shown to constitute a stationary solution of our present dynamical replica equations, suggests (at least to the left of the vertical dashed line, where replica symmetry should hold) that the time required for true equilibration diverges with $N$. Both the static RS replica equations and the long time limit of the dynamical RS replica equations represent distinct stationary solutions of the dynamical formalism, and the observed differences in $x(c)$ are manifestations of the non-commuting of the limits $N \rightarrow \infty$ and $t \rightarrow \infty$.

\section{Discussion}

In this paper, we have studied the sequential dynamics of finitely connected Ising spin models with random bonds, on Poissonian random graphs. Starting from the microscopic master equation we derived a dynamic equation for the joint spin-field probability distribution, which is exact in the infinite system size limit, but not closed. We then followed the usual prescriptions and assumptions of the dynamic replica theory [22] in order to close this equation. The result is a set of non-trivial coupled diffusion equations, in which the evaluation of the driving forces requires the solution of a saddle-point problem at each instance of time. The latter saddle-point equations are of a functional nature, and are derived within the replica-symmetric (RS) ansatz; they can be solved numerically by a conventional population dynamics algorithm [6].

As a first application, we have applied our dynamical theory to the dynamics of a simulated annealing algorithm (Glauber-type dynamics with a stepwise stochastic cooling schedule) when running to find a solution of the so-called minimal vertex cover (VC) problem [10], on finitely connected Poissonian random graphs. In this problem the local fields are essentially integer-valued, which simplifies our dynamical equations. We have derived dynamic equation for the joint probability of spins and non-negative integer fields. Upon solving the equations of our theory numerically and comparing the results with the outcome of numerical simulations of the algorithm, we find an excellent agreement between the theory and experiment. 
When compared to, e.g. the generating functional analysis method (GFA), the advantage of the dynamic replica theory (DRT) is that, unlike GFA, it does not give an effective number of scalar order parameters that grows exponentially with time. Although also in its present form $^{3}$, with the joint spin-field distribution as the core dynamical order parameter, the DRT method is not exact, it is certainly much more accurate than, e.g. any simple two-parameter theory [17], and can be systematically improved further by increasing the order parameter set [15], although at a numerical cost. We believe its wide applicability to be the main advantage of the dynamical theory presented in this paper. The formalism can be extended relatively easily to include, for instance, directed or non-Poissonian random graphs.

\section{Acknowledgments}

It is our great pleasure to thank I Pérez-Castillo, JPL Hatchett, A Annibale and M Weigt for interesting and helpful discussions.

\section{Appendix A. Averaging over disorder}

In this appendix, we give the details of the disorder averaging in equation (20) that brings us to equation (21). First, we rewrite slightly the term within the angular brackets, exploiting the symmetry of $c_{i j} J_{i j}$ under the index permutations $i \leftrightarrow j$ :

$$
\begin{aligned}
\langle\ldots\rangle_{\left\{c_{i j} J_{i j}\right\}} & =\left\langle c_{12} \delta\left[h-H_{2}^{1}+2 J_{12} \tilde{s}\right] \mathrm{e}^{-\mathrm{i} \sum_{\alpha i} \hat{h}_{i}^{\alpha} h_{i}\left(\sigma^{\alpha}\right)}\right\rangle_{\left\{c_{i j} J_{i j}\right\}} \\
& =\mathrm{e}^{-\mathrm{i} \theta \sum_{\alpha i} \hat{h}_{i}^{\alpha}}\left\langle c_{12} \delta\left[h-H_{2}^{1}+2 J_{12} \tilde{s}\right] \mathrm{e}^{-\mathrm{i} \sum_{i \neq j} c_{i j} J_{i j} \sum_{\alpha} \hat{h}_{i}^{\alpha} \sigma_{j}^{\alpha}}\right\rangle_{\left\{c_{i j} J_{i j}\right\}} \\
& =\mathrm{e}^{-\mathrm{i} \theta \sum_{\alpha i} \hat{h}_{i}^{\alpha}}\left\langle c_{12} \delta\left[h-H_{2}^{1}+2 J_{12} \tilde{s}\right] \mathrm{e}^{-\mathrm{i} \sum_{i<j} c_{i j} J_{i j} \sum_{\alpha}\left[\hat{h}_{i}^{\alpha} \sigma_{j}^{\alpha}+\hat{h}_{j}^{\alpha} \sigma_{i}^{\alpha}\right]}\right\rangle_{\left\{c_{i j} J_{i j}\right\}} .
\end{aligned}
$$

We then average over the connectivity disorder $\left\{c_{i j}\right\}$, which is defined by (3), followed by the bond disorder $\left\{J_{i j}\right\}$ :

$$
\begin{aligned}
\langle\ldots\rangle_{\left\{c_{i j} J_{i j}\right\}}= & \frac{c}{N} \mathrm{e}^{-\mathrm{i} \theta \sum_{\alpha i} \hat{h}_{i}^{\alpha}}\left\{\delta\left[h-H_{2}^{1}+2 J_{12} \tilde{s}\right] \mathrm{e}^{\left.-\mathrm{i} J_{12} \sum_{\alpha} \hat{h}_{1}^{\alpha} \sigma_{2}^{\alpha}+\hat{h}_{2}^{\alpha} \sigma_{1}^{\alpha}\right]}\right. \\
& \left.\times \prod_{i<j, j \neq 2}\left\{\frac{c}{N} \mathrm{e}^{-\mathrm{i} J_{i j} \sum_{\alpha}\left[\hat{h}_{i}^{\alpha} \sigma_{j}^{\alpha}+\hat{h}_{j}^{\alpha} \sigma_{i}^{\alpha}\right]}+\left(1-\frac{c}{N}\right)\right\}\right\rangle_{\left\{J_{i j}\right\}} \\
= & \frac{c}{N} \mathrm{e}^{-\mathrm{i} \theta \sum_{\alpha i} \hat{h}_{i}^{\alpha}} \int \mathrm{d} J P(J) \delta\left[h-H_{2}^{1}+2 J \tilde{s}\right] \mathrm{e}^{\left.-\mathrm{i} J \sum_{\alpha} \hat{h}_{1}^{\alpha} \sigma_{2}^{\alpha}+\hat{h}_{2}^{\alpha} \sigma_{1}^{\alpha}\right]} \\
& \times \prod_{i<j, j \neq 2}\left\{\frac{c}{N} \int \mathrm{d} J P(J) \mathrm{e}^{\left.-\mathrm{i} J \sum_{\alpha} \hat{h}_{i}^{\alpha} \sigma_{j}^{\alpha}+\hat{h}_{j}^{\alpha} \sigma_{i}^{\alpha}\right]}+1-\frac{c}{N}\right\} .
\end{aligned}
$$

Finally, we re-exponentiate the last line of the above expression, giving

$$
\begin{aligned}
\langle\ldots\rangle_{\left\{c_{i j} J_{i j}\right\}=} & \frac{c}{N} \\
& \mathrm{e}^{-\mathrm{i} \theta \sum_{\alpha i} \hat{h}_{i}^{\alpha}} \int \mathrm{d} J P(J) \delta\left[h-H_{2}^{1}+2 J \tilde{s}\right] \mathrm{e}^{-\mathrm{i} J \sum_{\alpha}\left[\hat{h}_{1}^{\alpha} \sigma_{2}^{\alpha}+\hat{h}_{2}^{\alpha} \sigma_{1}^{\alpha}\right]} \\
& \times \exp \left[\frac{c}{2 N} \sum_{i j}\left[\int \mathrm{d} J P(J) \mathrm{e}^{-\mathrm{i} J \sum_{\alpha}\left[\hat{h}_{i}^{\alpha} \sigma_{j}^{\alpha}+\hat{h}_{j}^{\alpha} \sigma_{i}^{\alpha}\right]}-1\right]+O(1)\right] .
\end{aligned}
$$

\footnotetext{
${ }^{3}$ For parallel stochastic dynamics, it can be shown that an exact formulation of DRT is possible for any discrete spin
} model, with an effective number of scalar order parameters that grows at most linearly with time [38]. 


\section{Appendix B. Calculation of the RS saddle-point equations}

We compute the RS versions of kernel (28) and the saddle-point equation (29). Assuming replica-symmetry transforms in these equations the averages over the effective measure, $\langle\ldots\rangle_{M} \rightarrow\langle\ldots\rangle_{M_{\mathrm{RS}}}$, with definition (35). In (28), (29) this gives

$$
\begin{aligned}
& D(s, h)=\frac{1}{M_{\mathrm{RS}}^{n}} \sum_{k \geqslant 0} \frac{c^{k}}{k !} \mathrm{e}^{-c} \int \prod_{\ell=1}^{k}\left\{\mathrm{~d} J_{\ell} P\left(J_{\ell}\right)\left\{\mathrm{d} P_{\ell}\right\} W\left[\left\{P_{\ell}\right\}\right]\right\} \\
& \times\left\{\sum_{\sigma_{1}} \int \mathrm{d} H_{1} \mathrm{~d} \hat{h}_{1} d\left(\sigma_{1}, H_{1}\right) \mathrm{e}^{\mathrm{i} \hat{h}_{1}\left[H_{1}-\theta\right]} \delta_{s, \sigma_{1}} \delta\left[h-H_{1}\right]\right. \\
& \left.\times \prod_{\ell=1}^{k}\left[\sum_{\sigma_{\ell}^{1}} \int \mathrm{d} \hat{h}_{\ell}^{1} P_{\ell}\left(\sigma_{\ell}^{1}, \hat{h}_{\ell}^{1}\right) \mathrm{e}^{-\mathrm{i} J_{\ell}\left[\hat{h}_{1} \sigma_{\ell}^{1}+\hat{h}_{\ell}^{1} \sigma_{1}\right]}\right]\right\} \\
& \times \prod_{\alpha=2}^{n}\left\{\sum_{\sigma_{\alpha}} \int \mathrm{d} H_{\alpha} \mathrm{d} \hat{h}_{\alpha} d\left(\sigma_{\alpha}, H_{\alpha}\right) \mathrm{e}^{\mathrm{i} \hat{h}_{\alpha}\left[H_{\alpha}-\theta\right]}\right. \\
& \left.\times \prod_{\ell=1}^{k}\left[\sum_{\sigma_{\ell}^{\alpha}} \int \mathrm{d} \hat{h}_{\ell}^{\alpha} P_{\ell}\left(\sigma_{\ell}^{\alpha}, \hat{h}_{\ell}^{\alpha}\right) \mathrm{e}^{-\mathrm{i} J_{\ell}\left[\hat{h}_{\alpha} \sigma_{\ell}^{\alpha}+\hat{h}_{\ell}^{\alpha} \sigma_{\alpha}\right]}\right]\right\} \\
& =\sum_{k \geqslant 0} \frac{c^{k}}{k !} \mathrm{e}^{-c} \int \prod_{\ell=1}^{k}\left\{\mathrm{~d} J_{\ell} P\left(J_{\ell}\right)\left\{\mathrm{d} P_{\ell}\right\} W\left[\left\{P_{\ell}\right\}\right]\right\} \frac{Z\left[\left\{P_{1}, \ldots, P_{k}\right\}\right]^{n-1}}{M_{\mathrm{RS}}^{n}} \\
& \times \sum_{\sigma} \prod_{\ell=1}^{k}\left[\sum_{\sigma_{\ell}} \int \mathrm{d} \hat{h}_{\ell} P_{\ell}\left(\sigma_{\ell}, \hat{h}_{\ell}\right) \mathrm{e}^{-\mathrm{i} J_{\ell} \hat{h}_{\ell} \sigma}\right] \\
& \times d\left(\sigma, \sum_{\ell=1}^{k} J_{\ell} \sigma_{\ell}+\theta\right) \delta_{s, \sigma} \delta\left[h-\sum_{\ell=1}^{k} J_{\ell} \sigma_{\ell}-\theta\right] \\
& =\sum_{k \geqslant 0} \frac{c^{k}}{k !} \mathrm{e}^{-c} \int \prod_{\ell=1}^{k}\left\{\mathrm{~d} J_{\ell} P\left(J_{\ell}\right)\left\{\mathrm{d} P_{\ell}\right\} W\left[\left\{P_{\ell}\right\}\right]\right\} \frac{Z\left[\left\{P_{1}, \ldots, P_{k}\right\}\right]^{n-1}}{M_{\mathrm{RS}}^{n}} \\
& \times d(s, h) \prod_{\ell=1}^{k}\left[\sum_{\sigma_{\ell}} \int \mathrm{d} \hat{h}_{\ell} P_{\ell}\left(\sigma_{\ell}, \hat{h}_{\ell}\right) \mathrm{e}^{-\mathrm{i} J_{\ell} \hat{h}_{\ell} s}\right] \delta\left[h-\sum_{\ell=1}^{k} J_{\ell} \sigma_{\ell}-\theta\right]
\end{aligned}
$$

and

$$
A\left[s, s^{\prime} ; h, h^{\prime} ; \tilde{s}\right]=\frac{\tilde{A}\left[s, s^{\prime} ; h, h^{\prime} ; \tilde{s}\right]}{\sum_{\sigma \sigma^{\prime}} \int \mathrm{d} H \mathrm{~d} H^{\prime} \tilde{A}\left[\sigma, \sigma^{\prime} ; H, H^{\prime} ; \tilde{s}\right]},
$$

where

$$
\begin{aligned}
\tilde{A}\left[s, s^{\prime} ; h, h^{\prime} ; \tilde{s}\right] & =\left\langle\delta_{s^{\prime}, \sigma_{1}} \delta_{s, \sigma_{1}^{\prime}} \delta\left[h^{\prime}-H_{1}\right] \delta\left[h-H_{1}^{\prime}+2 J \tilde{s}\right] \mathrm{e}^{-\mathrm{i} J\left[\hat{\boldsymbol{h}}_{\cdot} \cdot \boldsymbol{\sigma}^{\prime}+\hat{\boldsymbol{h}}^{\prime} \cdot \sigma\right]}\right\rangle_{J, M_{\mathrm{RS}}, M_{\mathrm{RS}}^{\prime}} \\
= & \frac{1}{M_{\mathrm{RS}}^{2 n}} \int \mathrm{d} J P(J) \sum_{\sigma \sigma^{\prime}} \int \mathrm{d} \boldsymbol{H} \mathrm{d} \boldsymbol{H}^{\prime} \mathrm{d} \hat{\boldsymbol{h}} \mathrm{d} \hat{\boldsymbol{h}}^{\prime} \delta_{s^{\prime}, \sigma_{1}} \delta_{s, \sigma_{1}^{\prime}} \delta\left[h^{\prime}-H_{1}\right] \delta\left[h-H_{1}^{\prime}+2 J \tilde{s}\right] \\
& \times \sum_{k \geqslant 0} \frac{c^{k}}{k !} \mathrm{e}^{-c} \int \prod_{\ell=1}^{k}\left\{\mathrm{~d} J_{\ell} P\left(J_{\ell}\right)\left\{\mathrm{d} P_{\ell}\right\} W\left[\left\{P_{\ell}\right\}\right]\right\}
\end{aligned}
$$




$$
\begin{aligned}
& \times \prod_{\alpha=1}^{n} d\left(\sigma_{\alpha}, H_{\alpha}\right) \mathrm{e}^{\mathrm{i} \hat{h}_{\alpha}\left[H_{\alpha}-\theta-J \sigma_{\alpha}^{\prime}\right]} \prod_{\ell=1}^{k}\left[\sum_{\sigma_{\ell}^{\alpha}} \int \mathrm{d} \hat{h}_{\ell}^{\alpha} P_{\ell}\left(\sigma_{\ell}^{\alpha}, \hat{h}_{\ell}^{\alpha}\right) \mathrm{e}^{-\mathrm{i} J_{\ell}\left[\hat{h}_{\alpha} \sigma_{\ell}^{\alpha}+\hat{h}_{\ell}^{\alpha} \sigma_{\alpha}\right]}\right] \\
& \times \sum_{m \geqslant 0} \frac{c^{m}}{m !} \mathrm{e}^{-c} \int \prod_{r=1}^{m}\left\{\mathrm{~d} J_{r} P\left(J_{r}\right)\left\{\mathrm{d} Q_{r}\right\} W\left[\left\{Q_{r}\right\}\right]\right\} \\
& \times \prod_{\alpha=1}^{n} d\left(\sigma_{\alpha}^{\prime}, H_{\alpha}^{\prime}\right) \mathrm{e}^{\mathrm{i} \hat{h}_{\alpha}^{\prime}\left[H_{\alpha}^{\prime}-\theta-J \sigma_{\alpha}\right]} \prod_{r=1}^{m}\left[\sum_{\sigma_{r}^{\alpha}} \int \mathrm{d} \hat{h}_{r}^{\alpha} Q_{r}\left(\sigma_{r}^{\alpha}, \hat{h}_{r}^{\alpha}\right) \mathrm{e}^{-\mathrm{i} J_{r}\left[\hat{h}_{\alpha}^{\prime} \sigma_{r}^{\alpha}+\hat{h}_{r}^{\alpha} \sigma_{\alpha}^{\prime}\right]}\right] \\
& =\frac{1}{M_{\mathrm{RS}}^{2 n}} \sum_{k \geqslant 0} \frac{c^{k}}{k !} \mathrm{e}^{-c} \int \prod_{\ell=1}^{k}\left\{\mathrm{~d} J_{\ell} P\left(J_{\ell}\right)\left\{\mathrm{d} P_{\ell}\right\} W\left[\left\{P_{\ell}\right\}\right]\right\} \\
& \times \sum_{m \geqslant 0} \frac{c^{m}}{m !} \mathrm{e}^{-c} \int \prod_{r=1}^{m}\left\{d J_{r} P\left(J_{r}\right)\left\{\mathrm{d} Q_{r}\right\} W\left[\left\{Q_{r}\right\}\right]\right\} \int \mathrm{d} J P(J) \\
& \times \sum_{\sigma \sigma^{\prime}} \prod_{\ell=1}^{k}\left[\sum_{\sigma_{\ell}} \int \mathrm{d} \hat{h}_{\ell} P_{\ell}\left(\sigma_{\ell}, \hat{h}_{\ell}\right) \mathrm{e}^{-\mathrm{i} J_{\ell} \hat{h}_{\ell} \sigma}\right] d\left(\sigma, \sum_{\ell=1}^{k} J_{\ell} \sigma_{\ell}+\theta+J \sigma^{\prime}\right) \\
& \times \prod_{r=1}^{m}\left[\sum_{\sigma_{r}} \int \mathrm{d} \hat{h}_{r} Q_{r}\left(\sigma_{r}, \hat{h}_{r}\right) \mathrm{e}^{-\mathrm{i} J_{r} \hat{h}_{r} \sigma^{\prime}}\right] d\left(\sigma^{\prime}, \sum_{r=1}^{m} J_{r} \sigma_{r}+\theta+J \sigma\right) \\
& \times \delta_{s, \sigma^{\prime}} \delta\left[h-\sum_{r=1}^{m} J_{r} \sigma_{r}-\theta-J \sigma+2 J \tilde{s}\right] \delta_{s^{\prime}, \sigma} \delta\left[h^{\prime}-\sum_{\ell=1}^{k} J_{\ell} \sigma_{\ell}-\theta-J \sigma^{\prime}\right] \\
& \times Z\left[\left\{P_{1}, \ldots, P_{k} \mid Q_{1}, \ldots, Q_{m}\right\}\right]^{n-1} .
\end{aligned}
$$

In the replica limit $n \rightarrow 0$ we get $\lim _{n \rightarrow 0} M_{\mathrm{RS}}^{n}=\lim _{n \rightarrow 0} Z[\ldots]^{n}=1$, and also $\lim _{n \rightarrow 0} \sum_{\sigma \sigma^{\prime}} \int \mathrm{d} H \mathrm{~d} H^{\prime} \tilde{A}\left[\sigma, \sigma^{\prime} ; H, H^{\prime} ; \tilde{s}\right]=1$. As a result, expressions (B.1) and (B.2) reduce to equations (39) and (40), respectively.

\section{Appendix C. Reduction of PDE to the system of ODEs}

In this appendix we show how the diffusion equation (16), written in terms of kernels (53) and (54), can be reduced to a system of ordinary differential equations. The discrete nature of fields (52) allows us to write (53) and (54) in terms of the probability distributions (64) and (65). Inserting (64) and (65) into both sides of (16) gives

$$
\begin{aligned}
\frac{\partial}{\partial t} \sum_{n \geqslant 0} P_{t}(s, n) & \delta(h-J n-\theta)=\frac{1}{2}[1+s \tanh [\beta h]] \sum_{n \geqslant 0} P_{t}(-s, n) \delta(h-J n-\theta) \\
& -\frac{1}{2}[1-s \tanh [\beta h]] \sum_{n \geqslant 0} P_{t}(s, n) \delta(h-J n-\theta) \\
& +\frac{1}{2} c \sum_{s^{\prime}} \int \mathrm{d} h^{\prime}\left[1-s^{\prime} \tanh \left[\beta h^{\prime}\right]\right] \sum_{n n^{\prime} \geqslant 0} A_{t}\left[s, s^{\prime} ; n, n^{\prime}\right] \\
& \times \delta\left[h^{\prime}-J\left(n^{\prime}+\delta_{s,-1}\right)-\theta\right] \delta\left[h-J\left(n+\delta_{s^{\prime},-1}+s^{\prime}\right)-\theta\right] \\
& -\frac{1}{2} c \sum_{s^{\prime}} \int \mathrm{d} h^{\prime}\left[1-s^{\prime} \tanh \left[\beta h^{\prime}\right]\right] \sum_{n n^{\prime} \geqslant 0} A_{t}\left[s, s^{\prime} ; n, n^{\prime}\right] \\
& \times \delta\left[h^{\prime}-J\left(n^{\prime}+\delta_{s,-1}\right)-\theta\right] \delta\left[h-J\left(n+\delta_{s^{\prime},-1}\right)-\theta\right] .
\end{aligned}
$$


In the left-hand side we move the time derivative inside the summation, and in the right-hand side we sum over $s^{\prime}$ and integrate over $h^{\prime}$. This leads to

$$
\begin{aligned}
\sum_{n \geqslant 0} \frac{\mathrm{d}}{\mathrm{d} t} P_{t}(s, n) & \delta(h-J n-\theta)=\sum_{n \geqslant 0} \delta(h-J n-\theta)\left\{\frac{1}{2}[1+s \tanh [\beta(J n+\theta)]] P_{t}(-s, n)\right. \\
& \left.-\frac{1}{2}[1-s \tanh [\beta(J n+\theta)]] P_{t}(s, n)\right\}+\frac{1}{2} c \sum_{n \geqslant 0} \delta(h-J n-\theta) \\
& \times \sum_{n^{\prime} \geqslant 0}\left\{\left[1+\tanh \left[\beta\left(J n^{\prime}+\theta+J \delta_{s,-1}\right)\right]\right] A_{t}\left[s,-1 ; n, n^{\prime}\right]\right. \\
& \left.-\left[1-\tanh \left[\beta\left(J n^{\prime}+\theta+J \delta_{s,-1}\right)\right]\right] A_{t}\left[s, 1 ; n, n^{\prime}\right]\right\}+\frac{1}{2} c \sum_{n \geqslant 1} \delta(h-J n-\theta) \\
& \times \sum_{n^{\prime} \geqslant 0}\left\{\left[1-\tanh \left[\beta\left(J n^{\prime}+\theta+J \delta_{s,-1}\right)\right]\right] A_{t}\left[s, 1 ; n-1, n^{\prime}\right]\right. \\
& \left.-\left[1+\tanh \left[\beta\left(J n^{\prime}+\theta+J \delta_{s,-1}\right)\right]\right] A_{t}\left[s,-1 ; n-1, n^{\prime}\right]\right\} .
\end{aligned}
$$

It follows from the above that the evolution in time of $P_{t}(s, n)$ is governed by (69).

\section{Appendix D. Initial conditions}

In this appendix we compute the values of the probability distribution $P(s, n)$, the functional distribution $\tilde{W}[\{\hat{P}\}]$ and the function $d(s, J n+\theta)$ at time $t=0$. We choose an initial state of the system in which all individual spin values are drawn randomly and independently, according to

$$
P_{0}(\sigma)=\prod_{i=1}^{N}\left\{\frac{1}{2}\left(1+m_{0}\right) \delta_{\sigma_{i} ; 1}+\frac{1}{2}\left(1-m_{0}\right) \delta_{\sigma_{i} ;-1}\right\},
$$

where $m_{0} \in[-1,1]$ is the prescribed initial magnetization. It follows that the joint spin-field distribution (53) at $t=0$ is given by

$$
\begin{aligned}
D_{0}(s, h) & =\lim _{N \rightarrow \infty} \sum_{\sigma} P_{0}(\sigma) \frac{1}{N} \sum_{i}^{N} \delta_{s, \sigma_{i}}\left\langle\delta\left[h-h_{i}(\sigma)\right]\right\rangle_{\left\{c_{i j}\right\}} \\
& =\frac{1}{2}\left(1+s m_{0}\right) \sum_{k \geqslant 0} \frac{c^{k}}{k !} \mathrm{e}^{-c} \prod_{\ell=1}^{k}\left\{\sum_{\sigma_{\ell}} \frac{1}{2}\left(1+\sigma_{\ell} m_{0}\right)\right\} \delta\left[h-J \sum_{\ell=1}^{k} \delta_{\sigma_{\ell},-1}-\theta\right] \\
& =\frac{1}{2}\left(1+s m_{0}\right) \sum_{n \geqslant 0} \frac{\left[\frac{1}{2} c\left(1-m_{0}\right)\right]^{n}}{n !} \exp \left[-\frac{1}{2} c\left(1-m_{0}\right)\right] \delta[h-J n-\theta] .
\end{aligned}
$$

The initial conditions for system (69) follow directly from the above expression:

$$
P_{0}(s, n)=\frac{1}{2}\left(1+s m_{0}\right) \frac{\left[\frac{1}{2} c\left(1-m_{0}\right)\right]^{n}}{n !} \exp \left[-\frac{1}{2} c\left(1-m_{0}\right)\right] .
$$

Furthermore, we see that the joint spin-field distribution (D.2) indeed takes the desired form of the saddle-point equation (61), with the functional distribution

$$
\tilde{W}[\{\hat{P}\}]=\prod_{\sigma, \sigma^{\prime}} \delta\left[\hat{P}\left(\sigma \mid J \delta_{\sigma^{\prime} ;-1}\right)-\frac{1}{2}\left(1+\sigma m_{0}\right)\right]
$$


and with

$$
d(s, J n+\theta)=\frac{1}{2}\left(1+s m_{0}\right) .
$$

It is a trivial matter to show that (D.4) and (D.2) are indeed the solutions of equation (61).

\section{Appendix E. Population dynamics}

The functional saddle-point equations (61) and (66) cannot, in general, be solved analytically (one trivial exception is the infinite temperature regime). We therefore resort to the so-called population dynamics algorithm [6] to obtain solutions numerically, solving equations (61) and (66) simultaneously for the functional distribution $\tilde{W}[\{\hat{P}\}]$ and the function $d(s, J n+\theta)$, given the (known) values of the probability distribution $P_{t}(s, n)$ at time $t$. We create a population of $\mathcal{N} 2 \times 2$ matrices $\hat{P}_{i}\left(\sigma \mid J \delta_{\sigma^{\prime},-1}\right)$, where $i=1, \ldots, \mathcal{N}$, and we initialize the numbers $d(s, J n+\theta)$, where $s \in\{-1,1\}$ and $n \in\{0,1,2, \ldots\}$. The initial values of population $\left\{\hat{P}_{i}\left(\sigma \mid J \delta_{\sigma^{\prime},-1}\right)\right\}$ and function $d(s, J n+\theta)$ are set to (D.4) and (D.2), respectively, at $t=0$, and for $t>0$ we simply reuse the values from the previous time step. We then execute an iterative process, whereby at each step we update the population of matrices and the numbers $d(s, J n+\theta)$ as follows:

(i) a number $k$ is drawn from the Poisson distribution $P_{c}(k)$ (3);

(ii) $k$ members $\hat{P}_{i}\left(\sigma \mid J \delta_{\sigma^{\prime},-1}\right)$ are selected randomly and independently from the population;

(iii) a new value for $P\left(\sigma \mid J \delta_{\sigma^{\prime},-1}\right)$ is calculated according to

$$
\hat{P}_{\text {new }}\left(\sigma \mid J \delta_{\sigma^{\prime},-1}\right)=\frac{\prod_{\ell=1}^{k}\left[\sum_{\sigma_{\ell}} \hat{P}_{\ell}\left(\sigma_{\ell} \mid J \delta_{\sigma,-1}\right)\right] d\left(\sigma, J \sum_{\ell=1}^{k} \delta_{\sigma_{\ell},-1}+\theta+J \delta_{\sigma^{\prime},-1}\right)}{\sum_{\sigma} \prod_{\ell=1}^{k}\left[\sum_{\sigma_{\ell}} \hat{P}_{\ell}\left(\sigma_{\ell} \mid J \delta_{\sigma,-1}\right)\right] d\left(\sigma, J \sum_{\ell=1}^{k} \delta_{\sigma_{\ell},-1}+\theta\right)} ;
$$

(iv) a member of the population is selected randomly, and replaced with the newly computed value $\hat{P}_{\text {new }}\left(\sigma \mid J \delta_{\sigma^{\prime},-1}\right)$

(v) a new function $d(s, J n+\theta)$ is computed according to

$$
\begin{aligned}
d_{\text {new }}(s, J n+\theta) & =P_{t}(s, n) \times\left[\sum_{k \geqslant 0} \frac{c^{k}}{k !} \mathrm{e}^{-c} \int \prod_{\ell=1}^{k}\left\{\left\{\mathrm{~d} \hat{P}_{\ell}\right\} \tilde{W}\left[\left\{\hat{P}_{\ell}\right\}\right]\right\}\right. \\
& \left.\times \frac{\prod_{\ell=1}^{k}\left[\sum_{\sigma_{\ell}} \hat{P}_{\ell}\left(\sigma_{\ell} \mid J \delta_{s,-1}\right)\right] \delta_{n, \sum_{\ell=1}^{k} \delta_{\sigma_{\ell},-1}}}{\sum_{\sigma} \prod_{\ell=1}^{k}\left[\sum_{\sigma_{\ell}} \hat{P}_{\ell}\left(\sigma_{\ell} \mid J \delta_{\sigma,-1}\right)\right] d\left(\sigma, J \sum_{\ell=1}^{k} \delta_{\sigma_{\ell},-1}+\theta\right)}\right]^{-1} .
\end{aligned}
$$

Here averaging over the functional measure $\tilde{W}$ is defined as averaging over the actual instantaneous population of $2 \times 2$ matrices. This iteration is repeated until the values of the function $d(s, J n+\theta)$ and the statistical properties of the population are stationary. The population measure $\tilde{W}$ will now be an estimate of the functional distribution (61), and the function $d(s, J n+\theta)$ is a fixed point of the iteration equation (E.2), i.e. a solution of our original saddle-point equation.

\section{References}

[1] Viana L and Bray A J 1985 J. Phys. C: Solid State Phys. 18 3037-51

[2] Mézard M, Parisi G and Virasoro M A 1987 Spin Glass Theory and Beyond (Singapore: World Scientific)

[3] Kanter I and Sompolinsky H 1987 Phys. Rev. Lett. 58 164-7

[4] Mézard M and Parisi G 1987 Europhys. Lett. 3 1067-74

[5] Monasson R 1998 J. Phys. A: Math. Gen. 31 513-29

[6] Mézard M and Parisi G 2001 Eur. Phys. J. B 20 217-33 
[7] Coolen A C C, Skantzos N S, Pérez-Castillo I, Perez Vicente C J, Hatchett J P L, Wemmenhove B and Nikoletopoulos T 2005 J. Phys. A: Math. Gen. 38 8289-317

[8] Monasson R and Zecchina R 1996 Phys. Rev. Lett. 76 3881-5

[9] Mézard M, Parisi G and Zecchina R 2002 Science 297 812-5

[10] Weigt M and Hartmann A K 2000 Phys. Rev. Lett. 84 6118-21

[11] Mulet R, Pagnani A, Weigt M and Zecchina R 2002 Phys. Rev. Lett. 89268701

[12] Zdeborova L and Krzakala F 2007 Phys. Rev. E 76031131

[13] Semerjian G and Cugliandolo L F 2003 Europhys. Lett. 61 247-53

[14] Semerjian G, Cugliandolo L F and Montanari A 2004 J. Stat. Phys. 115 493-530

[15] Semerjian G and Weigt M 2004 J. Phys. A: Math. Gen. 37 5525-46

[16] Hatchett J P L, Wemmenhove B, Pérez-Castillo I, Nikoletopoulos T, Skantzos N S and Coolen A C C 2004 J. Phys. A: Math. Gen. 37 6201-20

[17] Hatchett J P L, Pérez-Castillo I, Coolen A C C and Skantzos N S 2005 Phys. Rev. Lett. 95117204

[18] Hansen-Goos H and Weigt M 2005 J. Stat. Mech. Theory Exp. P08001

[19] Skantzos N S and Hatchett J P L 2007 Physica A 381 202-11

[20] de Dominics C 1978 Phys. Rev. B 18 4913-9

[21] Coolen A C C and Sherrington D 1994 J. Phys. A: Math. Gen. 27 7687-707

[22] Laughton S N, Coolen A C C and Sherrington D 1996 J. Phys. A: Math. Gen. 29 $763-86$

[23] Erdös P and Rényi A 1960 Publ. Math. Inst. Hung. Acad. Sci. 5 17-61

[24] Bedeaux D, Lakatos-Lindenberg K and Shuler K E 1971 J. Math. Phys. 12 2116-23

[25] Kühn R, van Mourik J, Weigt M and Zippelius A 2007 J. Phys. A: Math. Theor. 40 9227-52

[26] Garey M R and Johnson D S 1979 Computers and Intractability (San Francisco: Freeman)

[27] Gazmuri P G 1984 Networks 14 367-77

[28] Hartmann A K and Weigt M 2001 Theor. Comput. Sci. 265 199-225

[29] Frieze A M 1990 Discrete Math. 81 171-5

[30] Weigt M and Hartmann A K 2001 Phys. Rev. E 63056127

[31] De Bruijn N G 1981 Asymptotic Methods in Analysis (New York: Dover)

[32] Bauer M and Golinelli O 2001 Eur. Phys. J. B 24 339-52

[33] Zhou H J 2003 Eur. Phys. J. B 32 265-70

[34] Zhou H J 2005 Phys. Rev. Lett. 94217203

[35] Weigt M and Hartmann A K 2001 Phys. Rev. Lett. 86 1658-61

[36] Weigt M 2002 Eur. Phys. J. B 28 369-81

[37] Weigt M and Zhou H J 2006 Phys. Rev. E 74046110

[38] Coolen A C C and Guzai S D 2007 in preparation 